\title{
Dynamics of the Hungaria asteroids
}

\author{
Andrea Milani $^{\mathrm{a}}$, Zoran Kneževićc ${ }^{\mathrm{b}}$, Bojan Novaković ${ }^{\mathrm{b}}$, Alberto Cellino ${ }^{\mathrm{c}}$ \\ ${ }^{a}$ Dipartimento di Matematica, Università di Pisa, Largo Pontecorvo 5, 56127 Pisa, Italy \\ ${ }^{b}$ Astronomical Observatory, Volgina 7, 11060 Belgrade 38, Serbia \\ ${ }^{c}$ INAF-Osservatorio Astronomico di Torino Pino Torinese, Italy
}

\begin{abstract}
To try to understand the dynamical and collisional evolution of the Hungaria asteroids we have built a large catalog of accurate synthetic proper elements. Using the distribution of the Hungaria, in the spaces of proper elements and of proper frequencies, we can study the dynamical boundaries and the internal structure of the Hungaria region, both within a purely gravitational model and also showing the signature of the non-gravitational effects. We find a complex interaction between secular resonances, mean motion resonances, chaotic behavior and Yarkovsky-driven drift in semimajor axis. We also find a rare occurence of large scale instabilities, leading to escape from the region. This allows to explain the complex shape of a grouping which we suggest is a collisional family, including most Hungaria but by no means all; we provide an explicit list of non-members of the family. There are finer structures, of which the most significant is a set of very close asteroid couples, with extremely similar proper elements. Some of these could have had, in a comparatively recent past, very close approaches with low relative velocity. We argue that the Hungaria, because of the favourable observing conditions, may soon become the best known subgroup of the asteroid population.
\end{abstract}

Keywords: Asteroids, Asteroid dynamics, Collisional evolution, Nongravitational perturbations.

\section{The Hungaria region}

The inner edge of the asteroid main belt (for semimajor axes $a<2$ AU) has a comparatively populated portion at high inclination and low to

Email address: milani@dm.unipi.it (Andrea Milani) 


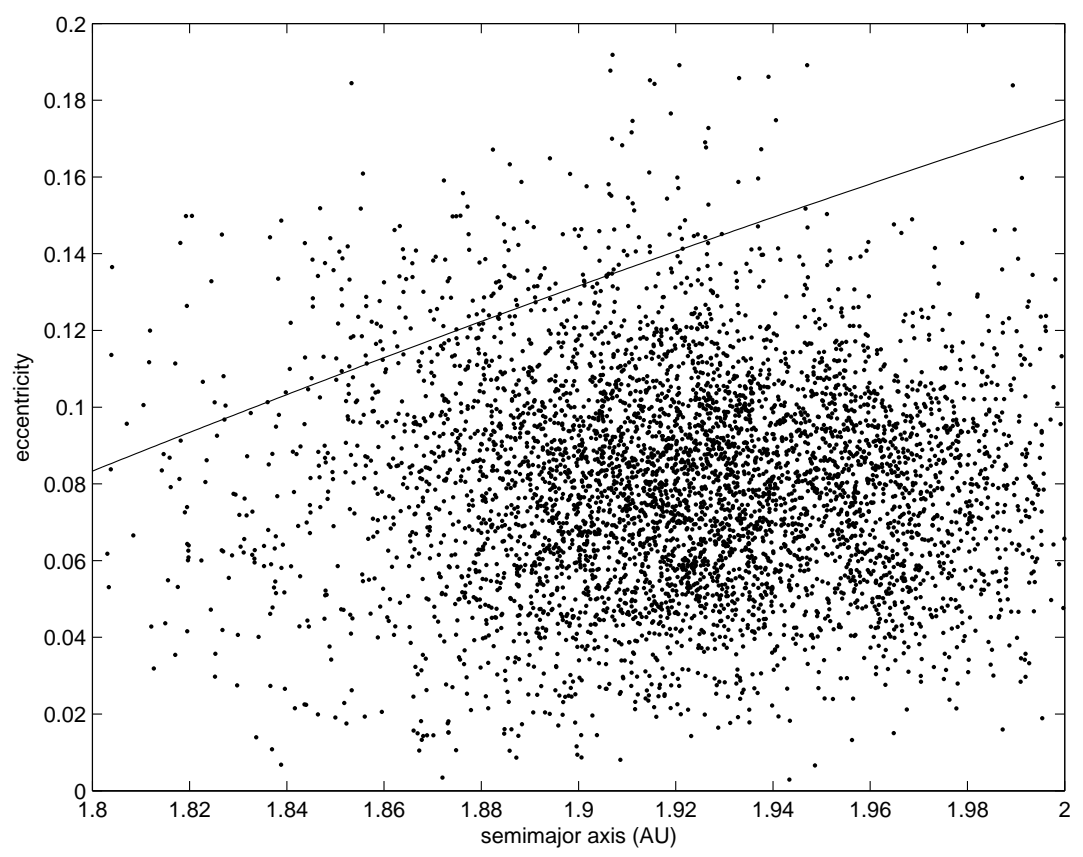

Figure 1: Osculating orbital elements semimajor axis and eccentricity for the 5025 Hungaria asteroids currently known and observed over multiple oppositions. In this plot we show 2477 numbered and 2548 multi-opposition Hungaria, selecting from the current catalogs (updated May 2009) the orbits with $1.8<a<2$ AU, $e<0.2$ and $I<30^{\circ}$. The line indicates where the perihelion would be at $1.65 \mathrm{AU}$, the aphelion distance of Mars.

moderate eccentricity: it is called the Hungaria region, after the first asteroid discovered which is resident there, namely (434) Hungaria. The limitation in eccentricity is allows for a perihelion large enough to avoid strong interactions with Mars, see Figure 1; the high inclination also contributes by keeping the asteroids far from the ecliptic plane most of the time, see Figure 2.

The other boundaries of the Hungaria region are less easy to understand on the basis of osculating elements only. However, a dynamical interpretation is possible in terms of secular perturbations, namely there are strong secular resonances, resulting from the perihelion of the orbit locked to the one of Jupiter, and to the node of the orbit locked to the one of Saturn. This, together with the Mars interaction, results in a dynamical instability boundary surrounding the Hungaria region from all sides, sharply separating 


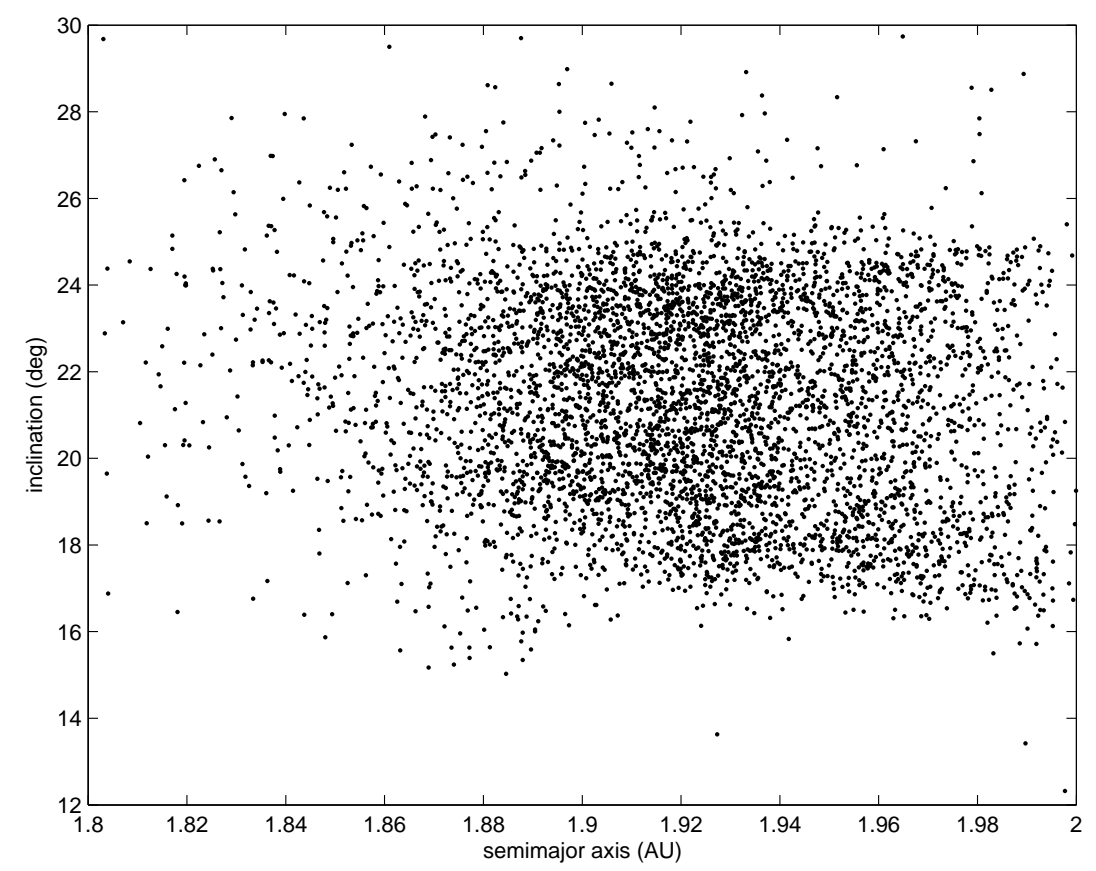

Figure 2: Osculating orbital elements semimajor axis and inclination for the same 5025 Hungaria asteroids of Figure 1.

it from the rest of the main belt.

This boundary can be crossed by asteroids leaking out of the Hungaria region, even considering only gravitational perturbations, but a small fraction of Hungaria are on orbits subject to this kind of unstable chaotic mechanisms. Thus the region is populated by asteroids which are, for the most part, not primordial but native to the region, that is fragments of a parent body which disrupted a long time ago.

The above argument fully explains the number density contrast between the Hungaria region and the surrounding gaps, regardless from the possible existence of an asteroid collisional family there. Still there is a family ${ }^{1}$, including (434) Hungaria, with strong evidence for a common collisional origin: this we call Hungaria family. It includes a large fraction of the Hungaria asteroids, but by no means all of them; tere is no firm evidence for other families

\footnotetext{
${ }^{1}$ As first proposed in [Lemaitre 1994].
} 
in the Hungaria region.

There are indications of internal structures inside the Hungaria family. These could belong to two types: sub-families and couples. A sub-family is a sub-group, of family asteroids more tightly packed than the surrounding members, which results from a breakup of a member of the original family at an epoch later, possibly much more recent, than the family formation event. A couple consists in just two asteroids, extremely close in the orbit space: the most striking property is that in some cases it is possible to find a very close approach of the two asteroids in a comparatively recent past, with a relative velocity at the closest approach much lower than the one expected for a collisional fragmentation.

All the above statements are not fundamentally new, in that many papers and presentations to meetings have argued along the same lines. The problem up to now was that there was no way to obtain a firm conclusion on many of these arguments and a rigorous proof of most statements about the Hungaria. This resulted from the fact that all sort of relevant data, such as proper elements, location of resonances, color indexes, rotation state information, constraints on physical properties such as density and thermal conductivity, where either lacking or inaccurate and inhomogeneous. To abuse of the information content of these low quality data to extract too many conclusions is a risky procedure. On the other hand there are indeed many interesting problems on which we would like to draw some robust conclusions.

When the present paper was almost complete, the paper [Warner et al. 2009] became available from Icarus online. This paper contains useful work, and the main results are in agreement with ours. However, many of the conclusions of that paper are weak for lack of accurate data: indeed the authors have made a great effort to make statements which can be reliable at least in a statistical sense. Thus we have found in this reading confirmation for the need of the work we have done for this paper, which is different in method and style more than in subject: our goal is to obtain rigorous results based on rigorous theories and high accuracy data. We will clearly state for which problems we have not been able to achieve this.

One reason for the difficulty met by all the previous authors who have worked on this subject was that one essential analytical tool was missing, namely good accuracy proper elements. Computations of proper elements for the Hungaria is especially difficult, for technical reasons explained in Section 2. However, we have found that the technique to compute synthetic proper elements, which we have developed in the past [Knežević and Milani 2000] 
and successfully used for the bulk of the main belt asteroids [Knežević and Milani 2003], is applicable to the Hungaria. Thus in this paper we first present in Section 2 our computation of proper elements, which are made available to the scientific community on our AstDyS web site ${ }^{2}$. Since the method to compute proper elements actually involves a comparatively long and accurate numerical integration of the orbits of all well known Hungaria, we also provide information on the accuracy and stability of the proper elements, on close approaches to Mars, on the Lyapounov exponents, and an explicit list of unstable cases.

Once the tool of proper elements is available, we can discuss in a quantitative way the dynamical structure (Section 3): stability boundaries, resonances and escape cases, signatures of non-gravitational perturbations. Then in Section 4 we establish the family classification, discussing escapers due to dynamical instabilities and the effects of non-gravitational perturbations. We also partially solve the problem of a rigorous listing of membership: this by using the only large and homogeneous data set of color information, that is SDSS MOC, in a way which is independent from the controversial color photometric taxonomies. In Section 5 we present the very close couples and investigate their dynamical and physical meaning.

The availability of our new tools does not imply that we can solve all the problems about the Hungaria: we would like to list three problems to which we give only very partial answers.

The first problem is how to identify the Hungaria region asteroids which belong to the Hungaria family. It is not possible to achieve this in all cases, because the orbital information needs to be complemented with physical information, too few of which is available. We can only show a method, list a number of proven non-members, and advocate the need for future data (see Section 4)

The second problem is the identification of sub-families inside the Hungaria family. Although it is possible to propose some of these, the currently available data do not allow firm conclusions. In particular the relationship, which should exist according to some models, between close couples and subfamilies can be neither proven nor contradicted (see Section 5). The situation should improve when much smaller Hungaria asteroids will be discovered.

The third problem is the quantitative modeling of the non-gravitational perturbations, especially the Yarkovsky effect. It is possible to show good

\footnotetext{
${ }^{2}$ http://hamilton.dm.unipi.it/astdys/
} 
evidence of secular drift in semimajor axis in the structure of the Hungaria family. However, to use in a quantitative way this information, e.g., for an accurate estimate of ages for both the family and the close couples, we would need to use other sources of information. These might come either from addtional observational constraints or from innovative methods of analysis of the existing data.

In Section 6 we discuss one reason why we think the Hungaria region is especially important to be studied, already now and much more in the near future. This is due to the possibility of reaching completeness in discoveries down to very small sizes, because of the particularly good observability conditions for the Hungaria in the context of the next generation surveys like Pan-STARRS and LSST. Finally, we summarize our conclusion in Section 7.

\section{Proper elements}

The computation of proper elements for the Hungaria asteroids would have been very difficult, if the only available method had been the analytic one. There are at least three reasons for this:

1. the inclination is high, thus analytical expansions with small parameters including $\sin I$ would fail;

2. the nearby linear secular resonances give rise small divisors, which introduce instabilities in the analytical computations; this applies in particular to the terms in the perturbations containing as argument the node of the Hungaria asteroid minus the node of Saturn, because the resonance occurs "below" the Hungaria region, at the same semimajor axes but lower inclination;

3. Mars is close, some Hungaria even have orbits crossing the one of Mars, and this results in divergence of most analytic expansions.

The first two difficulties could be overcome by using a semi-analytic theory, see [Lemaitre and Morbidelli 1994], but not the third one. The best option is to use a purely synthetic approach, based on data processing of the output from a long numerical integration. Note that also the values of the proper frequencies, and the location of the secular resonances, cannot be obtained with analytical methods based upon the expansion of the secular Hamiltonian in powers of eccentricity and inclination, such as the ones used in the free software secres we have made available on the AstDyS site. 


\subsection{Synthetic proper elements}

The procedure to compute synthetic proper elements for Hungaria asteroids is basically the same as the one used to compute the synthetic proper elements for the Main Belt asteroids. In the following we shall briefly describe the most important steps of the procedure, paying a special attention to details specific to the Hungaria asteroids.

The procedure to compute the asteroid synthetic proper elements consists of a set of purely numerical procedures, collectively called the synthetic theory. The procedure includes: (i) numerical integration of asteroid orbits in the framework of a suitable dynamical model; (ii) online digital filtering of the short periodic perturbations to compute the mean elements; (iii) Fourier analysis of the output to remove main forced terms and extract proper eccentricity, proper inclination, and the corresponding proper frequencies; for the proper semimajor axis, a simple average of the mean semimajor axis is used; (iv) check of the accuracy of the results by means of running box tests.

The numerical integration of asteroid orbits is performed by means of the public domain ORBIT9 software embedded in the multipurpose OrbFit package $^{3}$. The integrator employs as starter a symplectic single step method (implicit Runge-Kutta-Gauss), while a multi-step predictor performs most of the propagation [Milani and Nobili 1988]: this is possible because the eccentricity of the Hungaria orbits is moderate, while the high inclination does not matter for the truncation error.

The dynamical model used for the numerical integration is purely Newtonian and includes seven planets, from Venus to Neptune, as perturbing bodies. To account for the indirect effect of Mercury, its mass is added to the mass of the Sun and the barycentric correction is applied to the initial conditions [Milani and Knežević 1992]. Integrations for all asteroids initially covered $2 \mathrm{My}$, but have been extended to $10 \mathrm{My}$ for asteroids for which results from the short run turned out to be not accurate enough.

To generate numerical mean elements, that is asteroid elements freed from the short periodic perturbations, we made use of the on-line digital filter [Carpino et al. 1987, Knežević and Milani 2000]. In the 2 My integrations we set the decimation to 100 and output frequency to $200 \mathrm{y}^{-1}$, thus achieving nearly complete removal of the signal with periods up to 300 years.

The computation of synthetic proper elements includes removal of the

\footnotetext{
${ }^{3}$ Available from http://adams.dm.unipi.it/orbfit/
} 
forced secular perturbations, identification of the proper frequencies $n_{p}, g, s$ by fitting the time series of the corresponding arguments ${ }^{4}$, and extraction of proper modes by means of the Ferraz-Mello method [Ferraz-Mello 1981, Milani 1994]. Simultaneously the maximum LCE is estimated from a solution of the variational equation with random initial displacement, by the algorithm described in [Milani and Nobili 1992].

Finally, we perform running box tests for proper elements and proper frequencies, to assess their accuracy and stability in time. The tests consist in application of the same procedure to a number of shorter time intervals distributed over the entire span covered by integrations; these provide a set of distinct values of elements and frequencies allowing the computation of standard deviations. For the $2 \mathrm{My}$ integrations we used 11 boxes of length $\Delta T \simeq 1$ My. Note that here we made use of the dynamical model employed to compute proper elements for asteroids in the inner part of the Main Belt $(2.0<a<2.5 \mathrm{AU})$, but the time spans of the integrations were longer, actually the same as for the outer Main Belt asteroids $(2.5<a<4.9 \mathrm{AU})$. This was needed to remove the effects of many secular resonances, see Section 3.

We have extended the integration time span to $10 \mathrm{My}$ whenever the results obtained from the 2 My integrations proved to be insufficiently accurate or the orbits were found to be strongly chaotic. This time span is longer than the one used for inner main belt asteroids, but we considered this to be necessary to handle objects affected by higher order secular resonances, with periods of several million years. Thus, we repeated the computation of proper elements and frequencies whenever either $\sigma\left(a_{p}\right)>3 \times 10^{-4} \mathrm{AU}$ or $L C E>5 \times 10^{-5}$ $\mathrm{y}^{-1}$ (corresponding to Lyapounov time $T_{L}=20,000 \mathrm{yr}$; this accounts for chaotic orbits, due to mean motion resonances). Also, the same was done whenever either $\sigma\left(e_{p}\right)>3 \times 10^{-3}$ or $\sigma\left(\sin I_{p}\right)>2.5 \times 10^{-3}$ (to account for the orbits affected by secular resonances). The latter value is larger than the one used for the Main Belt $\left(1 \times 10^{-3}\right)$ in order to prevent too many asteroids in extended integrations (see Figure 5): obviously, given the much large inclinations, it is not possible to obtain an accuracy as good as most cases in the main belt. For the longer integrations the output frequency of the on-line filter was $500 \mathrm{y}^{-1}$, thus the signal with periods up to $750 \mathrm{y}$ was removed; 9 boxes of length $\Delta T \simeq 2$ My were used to compute standard

\footnotetext{
${ }^{4}$ The planetary frequencies are determined with essentially the same procedure on the output of the integration of planets over $20 \mathrm{My}$.
} 

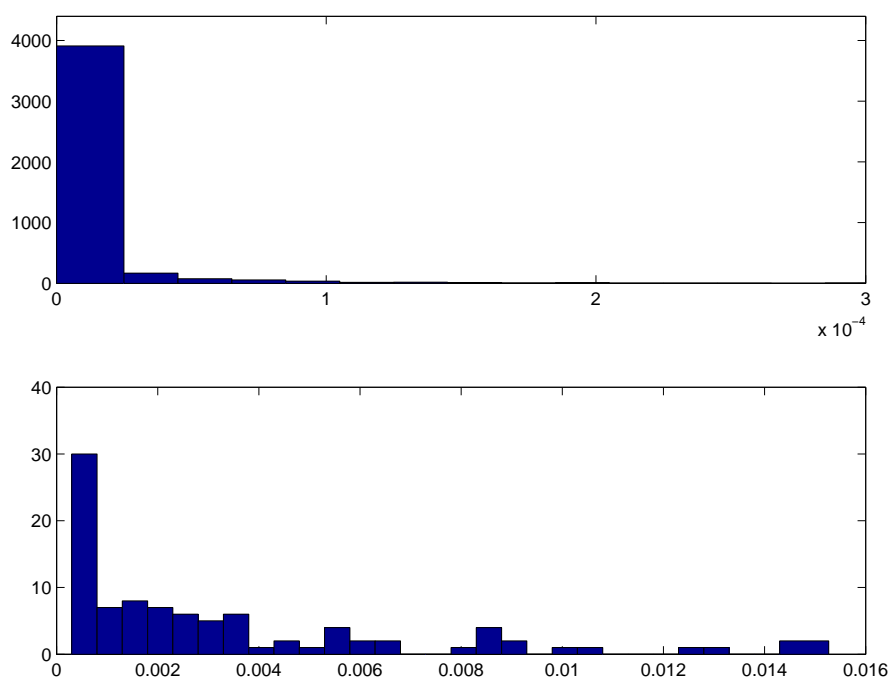

Figure 3: Number frequency distribution of standard deviations of proper semimajor axes below (top) and above (bottom) $3 \times 10^{-4} \mathrm{AU}$.

deviations of proper elements and frequencies.

\subsection{Accuracy of the proper elements}

The proper elements for a total of 4424 numbered and multi-opposition Hungaria asteroids are available from the AstDyS site ${ }^{5}$.

We have made an analysis of the overall performance of our procedure and of the accuracy of the results. In Figures 3, 4, 5 we plotted the number frequency distributions of the standard deviations of proper semimajor axes, eccentricities and (sine of) inclinations which give us a clear picture of the quality of the data we obtained. Each plot is divided in such a way that the upper panel contains histogram that covers a range of small to moderate values of standard deviation, while the bottom panel shows in more detail the distribution of the high value tail of the distribution.

As one can easily appreciate from these figures, for all three proper elements the vast majority of data is of very good accuracy. The histogram bins corresponding to the low values of the error in proper semimajor axis and proper eccentricity are by far the most populated ones. The situation is

\footnotetext{
${ }^{5}$ http://hamilton.dm.unipi.it/astdys/index.php?pc $=5$
} 

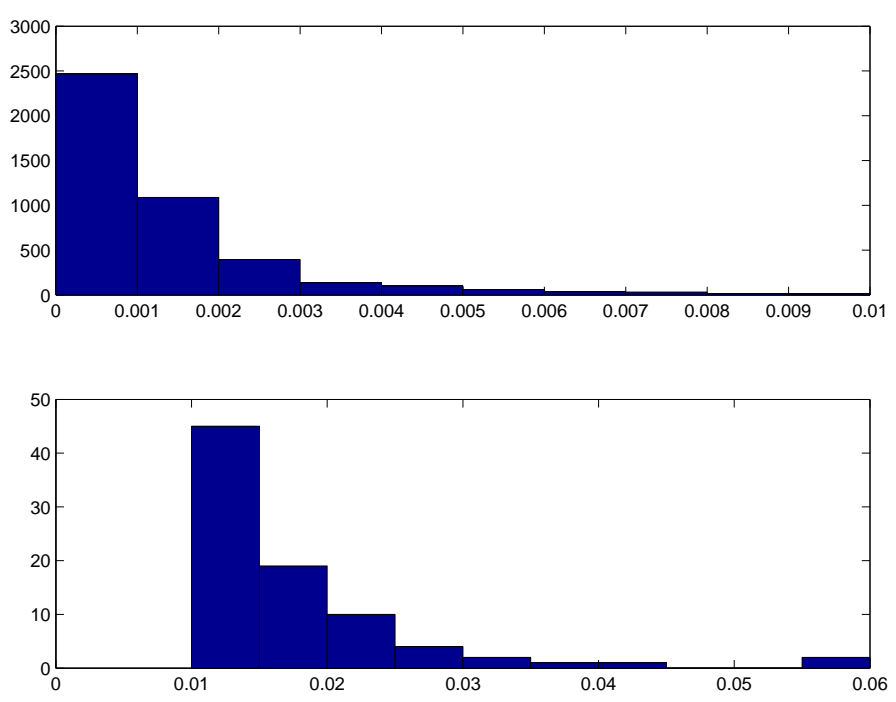

Figure 4: The same as Figure 3, but for standard deviations of eccentricities. Top: $\sigma\left(e_{p}\right)<10^{-2}$; Bottom: $\sigma\left(e_{p}\right)>10^{-2}$.

slightly different with the proper sine of inclination, where we observe somewhat larger spread of errors at the low end of the distribution. Here, however, we have to take into account that the inclinations of the Hungaria orbits are very high, so that these somewhat higher errors are still small with respect to the proper values themselves: in $99 \%$ of the cases, the $R M S(\sin I)<0.007$, that is the relative accuracy is less than 0.02 . This shows the overall good relative accuracy of our proper elements, which can be considered entirely appropriate for the study of the dynamical structure of the region and even of its finer substructures, as well as for dynamical family classification.

Really poor proper elements are found for only a rather small number of asteroids, of the order of $1-2 \%$ in each element (see the bottom panels of Figures 3, 4, 5). Many of these asteroids, however, typically have more than one low accuracy proper element, so that the number of distinct asteroids with poor elements is small. As we shall show in Section 3, the Hungaria region is crossed by several nonlinear secular resonances, giving rise to long periodic oscillations of the mean elements and affecting the determination of the corresponding proper values. 

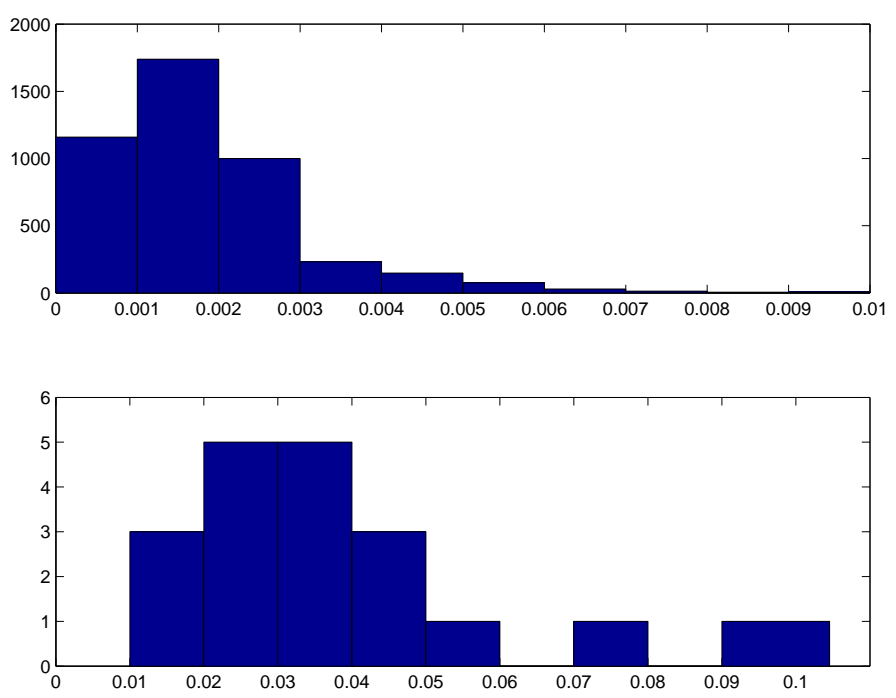

Figure 5: The same as Figure 3, but for standard deviations of (sine of) inclinations. Top: $\sigma\left(\sin I_{p}\right)<10^{-2}$; Bottom: $\sigma\left(\sin I_{p}\right)>10^{-2}$.

\subsection{Chaos and long term instability}

To assess the long term stability of motion of the Hungaria asteroids, and thus implicitly also of the proper elements we derived, we plot in Figure 6 the number frequency distribution of the Lyapounov Characteristic Exponents (LCEs) for ordered to moderately chaotic bodies (top panel) and for strongly chaotic ones (bottom panel). Again, there are several mean motion resonances in the region, giving rise to chaotic instabilities.

A number of bodies in the Hungaria region have strongly chaotic orbits, and for 25 of them we have identified repeated close approaches to Mars which appear to be the cause of the fast chaos (Lyapounov times are shorter than $5000 \mathrm{y}$ ). Note that the orbits being strongly chaotic, even the presence of close approaches to Mars is not a deterministic prediction, but something with a significant probability of happening to the real asteroid (whose initial conditions are not exactly the same as those available from the current orbit determination by AstDyS). The list of these bodies is given in Table 1 . 
Table 1: Strongly chaotic Hungaria, with close approaches to Mars. The columns give asteroid designation, osculating semimajor axis, eccentricity, inclination and perihelion distance obtained as final conditions for the integrations, that is for epoch $+10 \mathrm{My}$. Only one asteroid ended up in hyperbolic orbit in one of our test integrations.

\begin{tabular}{lccccc}
\hline Asteroid & $a[A U]$ & $e$ & $\left.I^{\circ}\right]$ & $q[A U]$ & Remarks \\
\hline 5641 & 1.6951 & 0.0675 & 25.014 & 1.5615 & \\
6141 & 1.9003 & 0.1461 & 19.882 & 1.5743 & \\
9068 & 1.8443 & 0.1352 & 24.590 & 1.5759 & \\
30935 & 1.9010 & 0.0426 & 30.253 & 1.6327 & \\
33888 & 1.9211 & 0.1454 & 14.933 & 1.6792 & \\
42811 & 1.8285 & 0.0920 & 27.462 & 1.6129 & \\
123597 & 1.7597 & 0.1986 & 31.067 & 1.6318 & \\
134746 & 1.8846 & 0.1556 & 19.166 & 1.5941 & \\
139798 & 1.9150 & 0.2103 & 18.175 & 1.5824 & \\
149809 & 1.8619 & 0.1049 & 20.560 & 1.6837 & \\
1996DC2 & 1.7592 & 0.3598 & 32.766 & 1.7156 & \\
1999RJ41 & 1.8102 & 0.1285 & 25.263 & 1.5579 & \\
2002AA22 & 1.8697 & 0.1203 & 23.491 & 1.6386 & \\
2002GG2 & 1.9631 & 0.0534 & 4.707 & 1.7349 & \\
2002OP28 & 1.8525 & 0.1436 & 21.216 & 1.5752 & \\
2002RN137 & 1.8831 & 0.1817 & 23.893 & 1.5946 & \\
2002TP161 & 1.9000 & 0.1596 & 16.158 & 1.6046 & \\
2003MW1 & 1.8790 & 0.1796 & 23.062 & 1.6678 & \\
2004GO28 & 1.9745 & 0.2341 & 3.874 & 1.7219 & \\
2004LL18 & 1.9433 & 0.1460 & 23.689 & 1.7014 & \\
2004PW39 & 1.8696 & 0.1169 & 21.671 & 1.7040 & \\
2005QY175 & 1.8881 & 0.1351 & 23.608 & 1.6406 & hyperbolic \\
2005TL15 & 1.8414 & 0.1404 & 23.541 & 1.6010 & \\
2007AY19 & 1.9156 & 0.1935 & 7.583 & 1.6133 & \\
2007YP13 & 1.8199 & 0.1454 & 16.788 & 1.6565 & \\
\hline & & & & & \\
& & & & &
\end{tabular}



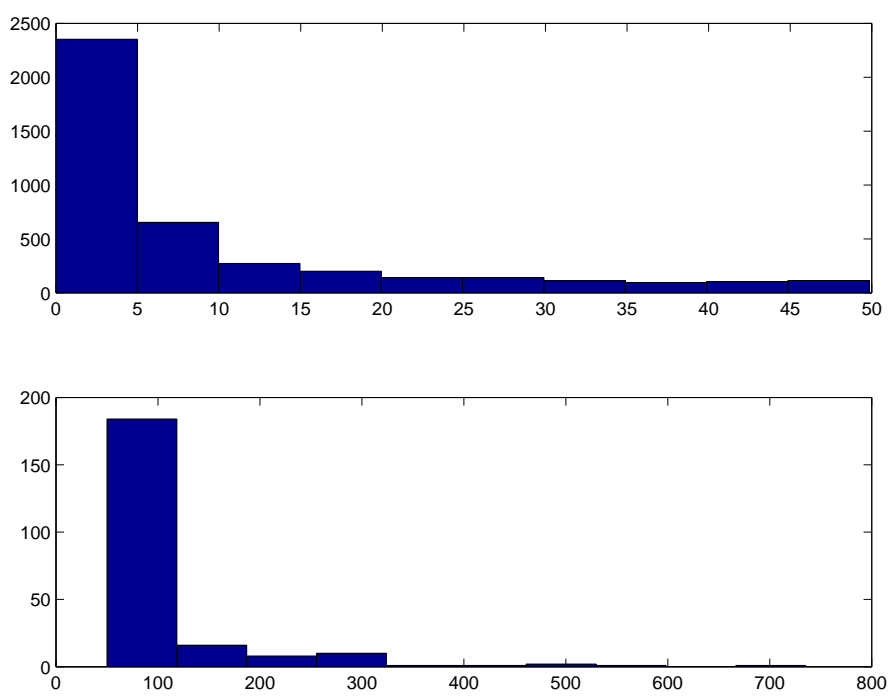

Figure 6: Number frequency distribution of Lyapounov Characteristic Exponents below (top) and above (bottom) $5 \times 10^{-5} \mathrm{y}^{-1}$; the unit on the abscissa is $10^{-6}$ $\mathrm{y}^{-1}$.

\section{Dynamical structure and stability}

Let us now observe the Hungaria region in the proper elements space, and in the space of proper frequencies; moreover, we have available other byproducts of the numerical integrations, such as LCE, the occurrence of close approaches, and even the detailed output orbit by orbit to analyze specially interesting cases.

\subsection{Dynamical boundaries}

The question is whether the obvious gaps with extremely reduced number densities, separating the Hungaria region from neighboring regions populated by asteroids, are dynamical boundaries, that is regions of instability. In fact, by using the proper frequencies $g$ (average rate of the perihelion longitude $\varpi$ ) and $s$ (average rate of the node longitude $\Omega$ ), we can identify the main secular resonances in and near the Hungaria region.

From the plot of the Hungaria asteroids in the $(g, s)$ plane (see Figure 7 ) we see that the main secular resonances, the ones already appearing in the linear secular perturbation theories, are bounding the Hungaria region on all four sides of the plot: the resonant values are $g=g_{5}=4.25 \operatorname{arcsec} / \mathrm{y}$ on 


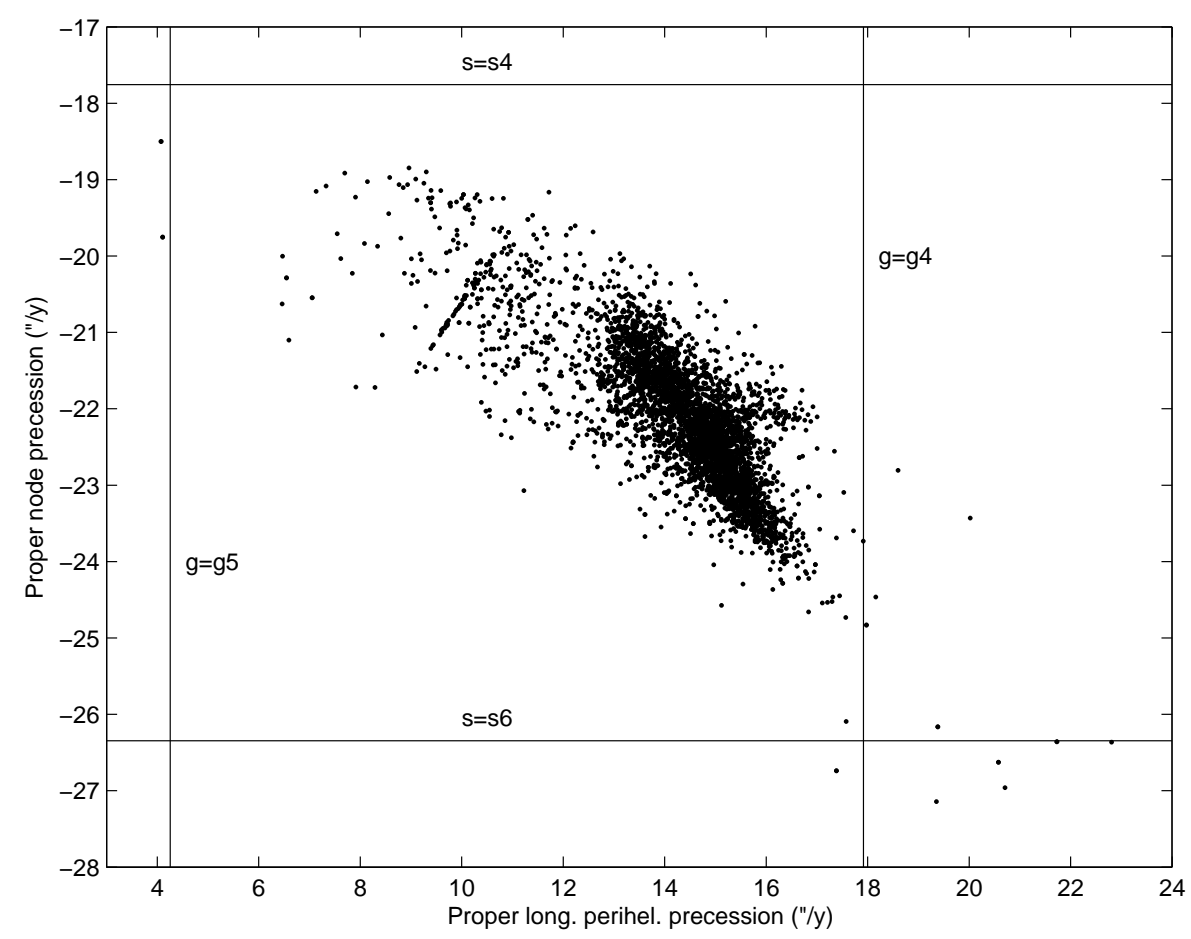

Figure 7: The proper frequencies $g$ and $s$ of the Hungaria, in arcsec/y. Among the secular resonances described in the text, $s-s_{6}$ affects few objects in the lower right corner, $g-g_{5}$ only two isolated objects on the upper left corner.

the left, $g=g_{4}=17.92$ on the right, $s=s_{6}=-26.35$ at the bottom, and $s=s_{4}=-17.76$ at the top of the figure ${ }^{6}$. The $g-g_{6}$ resonance cannot affect directly the Hungaria region, because the resonant value $g=28.25$ occurs for a semimajor axis $>2 \mathrm{AU}$, and the mean motion resonances $2 / 3$ with mars and $4 / 1$ with Jupiter are in between.

The secular resonances with the smaller planet Mars are also significant because Mars is close and also has a comparatively large inclination ${ }^{7}$; thus the cause of the gap for larger $s$ is the $s-s_{4}$ resonance. As an example, (76802) $2000 \mathrm{PV}_{27}$ has $s=-18.9 \operatorname{arcsec} / \mathrm{y}$, that is $s$ is close to $s_{3}$, but shows

\footnotetext{
${ }^{6}$ Secular frequencies for the inner planets are from [Laskar et al. 2004].

${ }^{7}$ The inclination of the Earth, even when considered with respect to the invariant plane, is much less than that of Mars.
} 


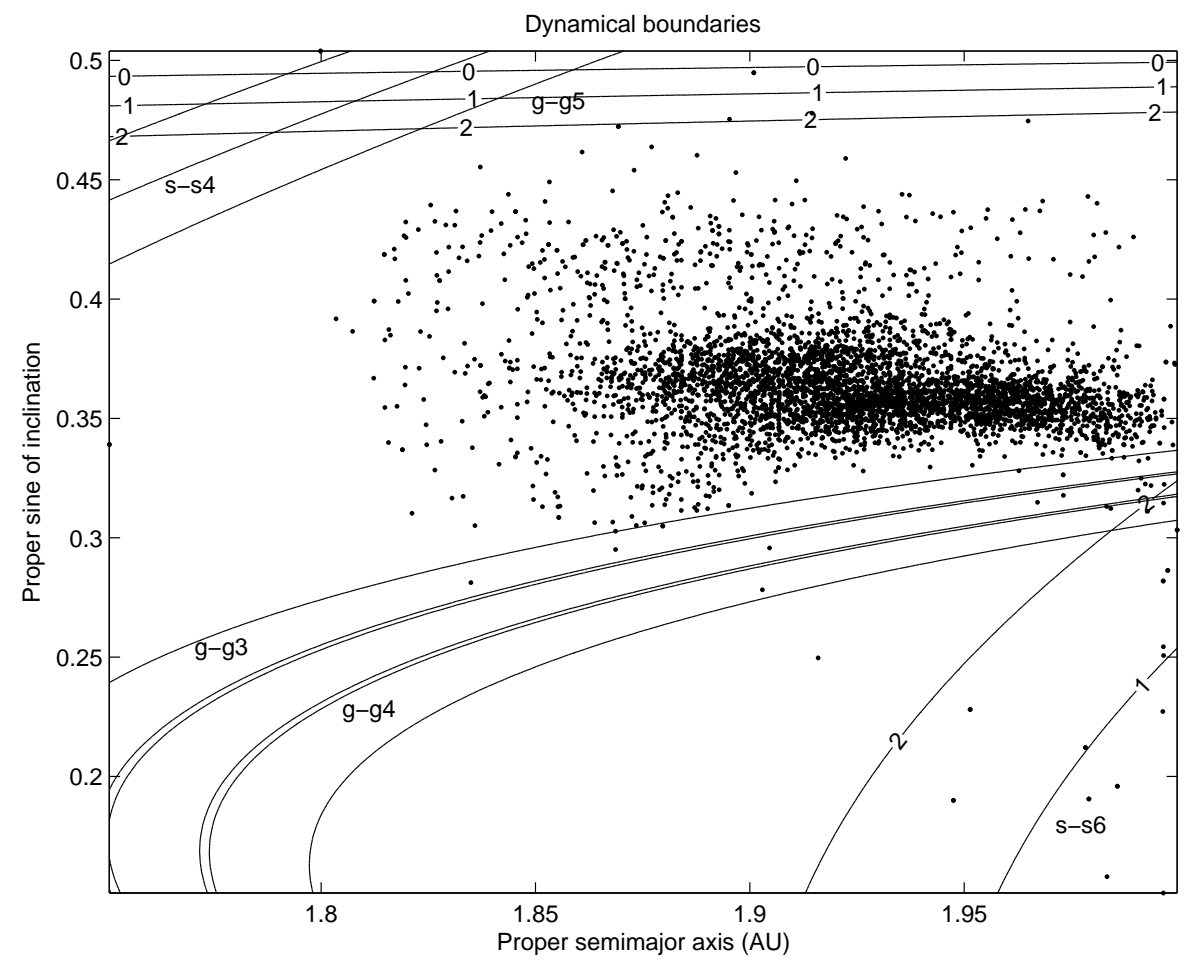

Figure 8: The Hungaria asteroids projected on the proper semimajor axis/proper sine of inclination plane. We have also drawn contour lines (labeled with the corresponding value) for the small divisors associated to the secular resonances $g-g_{6}$ and $g-g_{5}$, and contour lines for the values $-0.5,0,+0.5 \operatorname{arcsec} / \mathrm{y}$ for the secular resonances $g-s-g_{5}+s_{6}, 2 s-s_{6}-s_{4}$ and $g-2 s-g_{5}+2 s_{6}$.

oscillations of the inclination with two dominant periods corresponding to $s-s_{6}$ and $s-s_{4}$.

The proper inclination $I_{p}$ has a somewhat lower spread than the osculating inclination, with an evident dense core between $20^{\circ}$ and $23^{\circ}$ (see Figure 8$)^{8}$. In the $\left(a_{p}, \sin I_{p}\right)$ plane the Hungaria region appears to have very well defined boundaries.

The synthetic method for the computation of proper elements, unlike the

\footnotetext{
${ }^{8}$ Note that these values are not directly comparable with those of [Warner et al. 2009], because they do use long term averages, not proper elements.
} 
analytical one, does not provide directly the locations of the secular resonances in the proper elements space. However, there is an indirect way to compute such locations, as used in [Milani 1994]. We can use the secular frequencies $g$ and $s$, computed for each Hungaria with the synthetic method, to fit a smooth model of these frequencies. We have used a polynomial model obtained as a Taylor series, centered at the values of the proper elements for (434) Hungaria $\left(a_{H}=1.9443 e_{H}=0.0779 \sin I_{H}=0.3562\right)$, and truncated to degree 2. That is, we have used the differences $x=e_{p}-e_{H}, y=$ $\sin I_{p}-\sin I_{H}, z=a_{p}-a_{H}$ ), then normalized by dividing by the standard deviations of each of them $(0.0210,0.0243,0.0412$, respectively) obtaining the variables $\hat{x}, \hat{y}, \hat{z}$. The model which has been fitted had as base functions $1, \hat{x}, \hat{y}, \hat{z}, \hat{x}^{2}, \hat{y}^{2}, \hat{z}^{2}, \hat{x} \hat{y}, \hat{x} \hat{z}, \hat{y} \hat{z}$. The fit coefficients are given in Table 2 .

Table 2: Fit coefficients for the synthetic proper frequencies. For each base function:in column 1, the coefficients for $g$ and $s$ are in columns 2 and 3, respectively.

\begin{tabular}{crr}
\hline base function & $g(\operatorname{arcsec} / \mathrm{y})$ & $s(\operatorname{arcsec} / \mathrm{y})$ \\
\hline 1 & 15.1286 & -22.6719 \\
$\hat{x}$ & -0.0077 & -0.2459 \\
$\hat{y}$ & -1.4199 & 0.4203 \\
$\hat{z}$ & 0.4304 & -0.8095 \\
$\hat{x}^{2}$ & -0.0075 & -0.0315 \\
$\hat{y}^{2}$ & -0.0761 & 0.0166 \\
$\hat{z}^{2}$ & 0.0011 & -0.0155 \\
$\hat{x} \hat{y}$ & 0.0341 & -0.0067 \\
$\hat{x} z$ & -0.0134 & -0.0081 \\
$\hat{y} \hat{z}$ & -0.0587 & 0.0136 \\
\hline
\end{tabular}

This fit provides a model for the changes in the proper secular frequencies across the Hungaria region, and by extrapolation also somewhat outside (provided no major resonance is crossed). This model is approximate: the RMS of the residuals (synthetic values of the frequencies minus model values) is 0.13 and 0.06 arcsec/yr for $g$ and $s$, respectively. However, these uncertainties are much less than the width of the secular resonances we are interested in, and comparable to the uncertainty of the synthetic frequencies as computed with the running box method. As it is clear from the Table, as well as from the intuitive idea of weaker and stronger interaction with Jupiter, the frequency $g$ of precession of the perihelion decreases for decreasing $a_{p}$ and for increasing $\sin I_{p}$; the frequency $s$ of precession of the node increases (thus decreases in absolute value) for decreasing $a_{p}$ and for increasing $\sin I_{p}$. The 
value of $e_{p}$ has less effect on the secular frequencies (because $e_{p}$ is smaller than $\sin I_{p}$ in this region).

We have superimposed in Figure 8 to the values of the proper elements for the Hungaria the lines delineating the location of the main secular resonances which can be responsible for the dynamical boundaries of the region. The $g-g_{5}$ resonance is just above the Hungaria (for $I_{p}>29^{\circ}$ ), $s-s_{6}$ is below on the right. On the left for higher $I_{p}$ there is the $s-s_{4}$ resonance. The curved boundary below the dense core should be due to $g-g_{3}$ and $g-g_{4}$ resonances, see [Michel and Froeschlé 1997, Figure 2]; it is not easy to decide which of the two is more important, but anyway the two together are a dynamical boundary.

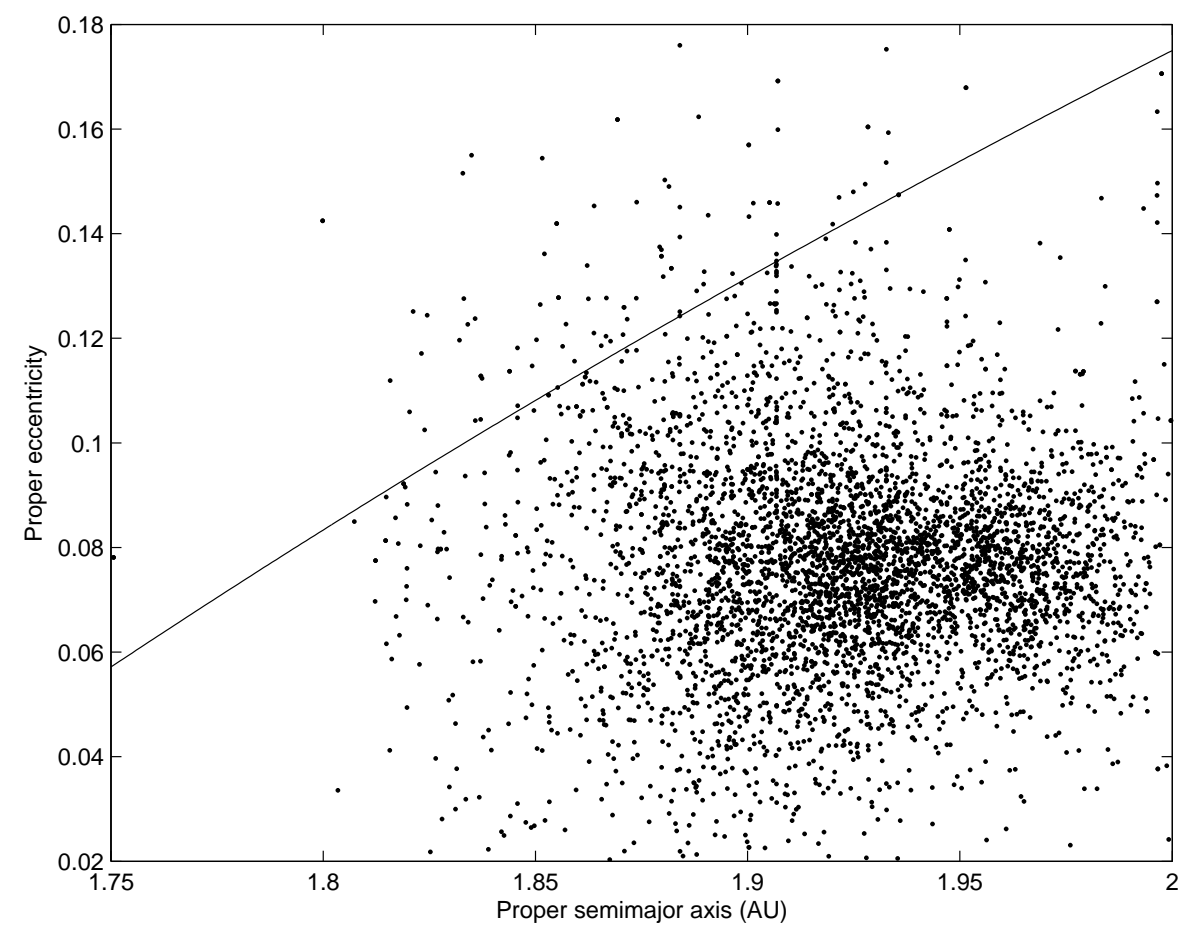

Figure 9: The Hungaria asteroids projected on the proper semimajor axis/proper eccentricity plane. We have also drawn a line corresponding to $a_{p}\left(1-e_{p}\right)=1.65$ $\mathrm{AU}$, which corresponds to the current aphelion distance of Mars.

There are some objects in the $s-s_{6}$ resonance, in the lower right corner of Figure 8, e.g., (33888) $2000 \mathrm{KG}_{21}$, for which the secular growth of the 
eccentricity leads to Mars crossing (see Table 1). Very few Hungaria can be locked in the $g-g_{5}$ resonance, we have found just two: (30935) Davasobel and $1996 \mathrm{DC}_{2}$, with unstable orbits leading also to Mars crossing (Table 1).

The proper eccentricity $e_{p}$ exhibits a similar core between 0.05 and 0.1 , but with a less sharp drop of density beyond the core boundary (see Figure 9 ). We have plotted on Figure 9 the same "Mars crossing" line $a_{p}\left(1-e_{p}\right)=1.65$ $\mathrm{AU}$, indicating that the average perihelion is at the aphelion of Mars. Objects beyond that line are Mars crossers most of the time, but not always.

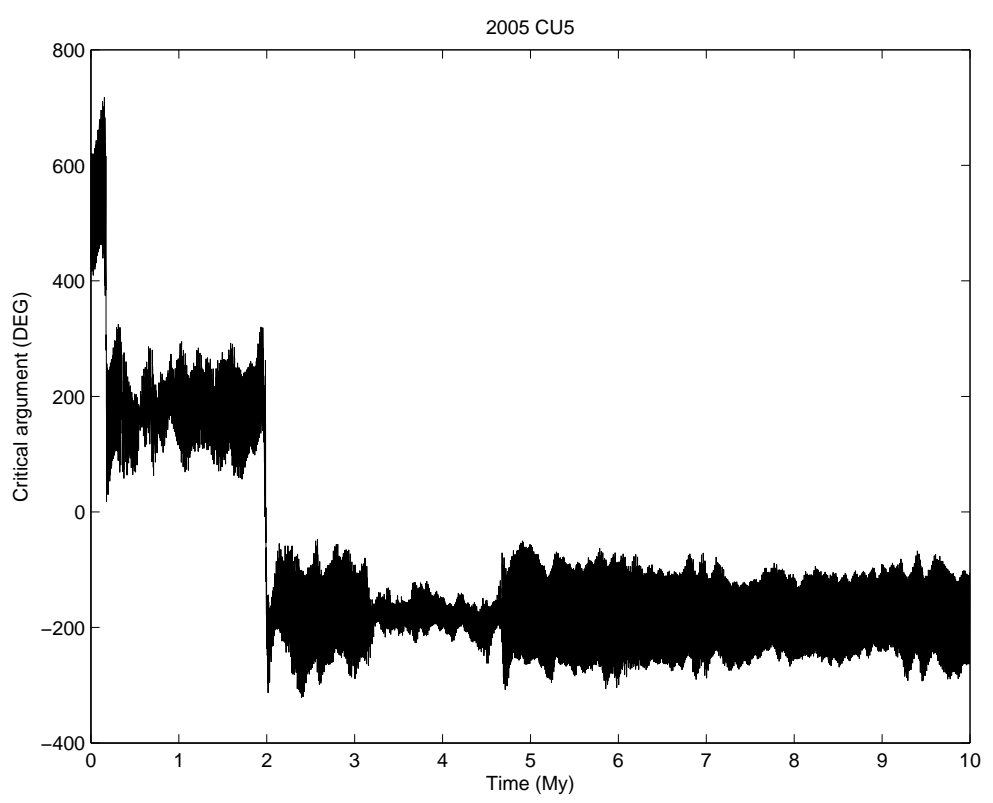

Figure 10: The critical argument of the $2 / 3$ mean motion resonance with Mars for $2005 \mathrm{CU}_{5}$ is locked in libration, with only a few cycle slips over $10 \mathrm{My}$.

The proper semimajor axes are distributed between 1.8 and 2 AU, with just one exception (2002 $\mathrm{AA}_{22}$ ); however, the distribution is obviously asymmetric, with much more objects for $a>1.87 \mathrm{AU}$. The outer boundary is controlled by the $s-s_{6}$ secular resonance (especially for low $I_{p}$ ) together with the $2 / 3$ mean motion resonance with Mars, the later as proposed by [Warner et al. 2009]. The effect of the mean motion resonance can be seen as a line of objects with larger eccentricities on the right hand side border of Figure 9: as an example, $2005 \mathrm{CU}_{5}$ will have the argument $3 \lambda-2 \lambda_{4}-\varpi$ in libration for most of the time in the next $10 \mathrm{My}$, see Figure 10. The $5 / 1$ mean 
motion resonance with Jupiter occurs for semimajor axis $<1.8$ AU, thus it is not the inner boundary, which is entirely due to the instability resulting from deep Mars crossing.

In conclusion, the Hungaria region has natural dynamical boundaries, where strong instabilities, arising from either secular resonances with Jupiter, Saturn and Mars or close approaches to Mars, depopulate leaving large gaps in the phase space.

\subsection{Resonances inside the Hungaria region}

To identify critical locations in the phase space where resonances affect the stability of the proper elements we can use the standard deviations of the synthetic proper elements, as computed with the running box method. In Figure 11 we plot the Hungaria with moderately unstable $e_{p}$ or $\sin I_{p}$. This shows clearly at least three features, which could be associated with the nonlinear secular resonances $g-s-g_{5}+s_{6}, 2 s-s_{6}-s_{4}$ and $g-2 s-g_{5}+2 s_{6}$; the first two are of degree 4 , that is arise from terms in the secular Hamiltonian of degree at least 4 in eccentricity and inclination [Milani and Knežević 1990, Knežević et al. 1991], the third one is of degree 6.

For example, we investigate the strongest of these resonances which, on the basis of the order and mass of the planets involved, should be $g-s-g_{5}+s_{6}$ : objects with the critical argument $\varpi-\Omega-\varpi_{5}+\Omega_{6}$ in libration can be easily found, as an example (43369) $2000 \mathrm{WP}_{3}$, see Figures 12 and 13, showing a term in eccentricity with period $\simeq 3.3 \mathrm{My}$ and an amplitude $\simeq 0.023$. The corresponding periodic terms in inclination have amplitude $\simeq 0.25^{\circ}$. This resonance occurs for $I_{p}$ around $25^{\circ}$, that is at inclinations higher than the ones of the dense core (that is, the core of the family described in the next section).

The presence of the nonlinear resonance $2 s-s_{6}-s_{4}$ with argument containing the node of Mars is not surprising, because of the important role played by the relative position of the orbital planes in decreasing the interaction. However, to prove that this resonance is responsible for the observed instability of the proper elements is not easy, because of the complex behavior of Mars inclination and longitude of node (with the inclination with respect to the invariable plane occasionally passing through zero). Thus we have resorted to a negative proof: we have recomputed the orbits of the Hungaria with node frequency $-22.5<s<-21.5$ arcsec/y in a dynamical model without Mars, and found that the increased instability of proper elements arcsec/y does not occur at all around $\left(s_{4}+s_{6}\right) / 2=-22.05$. Thus the 


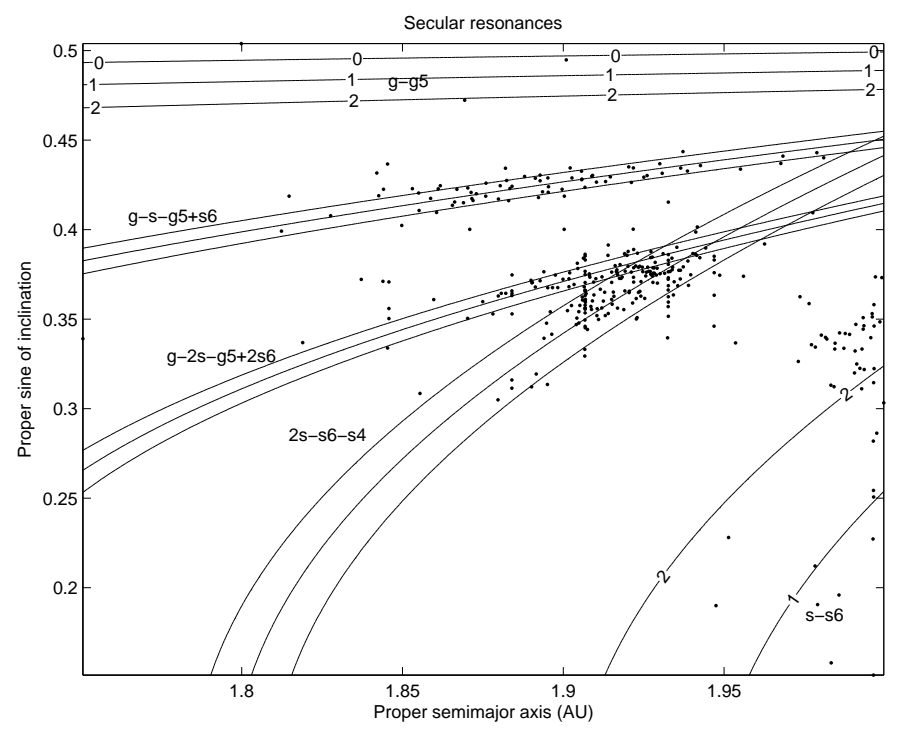

Figure 11: The proper elements $a_{p}$ and $\sin I_{p}$ are plotted only if they have a significant instability, with standard deviation for either $e_{p}$ or $\sin I_{p}$ above $5 \times 10^{-3}$. The contour lines are for the small divisors of the secular resonances discussed in the text.

resonance which causes the instability must contain the secular frequencies of Mars, and the one we have proposed is the lowest degree one.

The nonlinear secular resonances do not result, by themselves, in a significantly increased dispersion of the proper orbital elements, because these resonances are effective only on narrow bands within the Hungaria region.

The Hungaria region is also crossed by many mean motion resonances, which appear as channels within a narrow range of $a_{p}$ along which $e_{p}, I_{p}$ can diffuse. This is particularly evident in the dense core corresponding to the Hungaria family, and is the same phenomenon already known for main belt asteroid families [Knežević and Milani 2000, Figures 10 and 11]. A clear example of this, for $a_{p} \simeq 1.9068 \mathrm{AU}$, is shown in Figure 14 .

We have selected as example the asteroid $2001 \mathrm{TH}_{13}$, which should in the past have been in the Hungaria dense core, while now its osculating eccentricity can reach deep Mars-crossing levels (up to 0.22). Figure 15 shows the large oscillations in the mean semimajor axis (filtered in such a way that periods up to $300 \mathrm{y}$ are removed) over the last $2 \mathrm{My}$. At least 3 


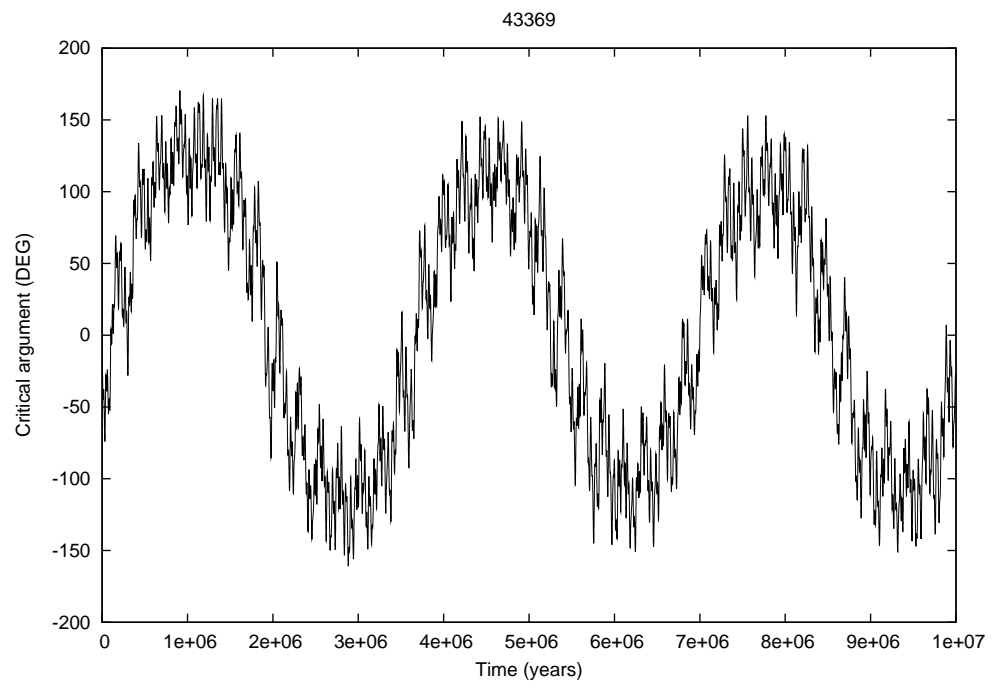

Figure 12: The critical argument of the resonance $g-s-g_{5}+s_{6}$ for the asteroid (43369) $2000 \mathrm{WP}_{3}$, propagated over $10 \mathrm{My}$. A high amplitude libration with a period of $\simeq 3.3 \mathrm{My}$ is clearly visible.

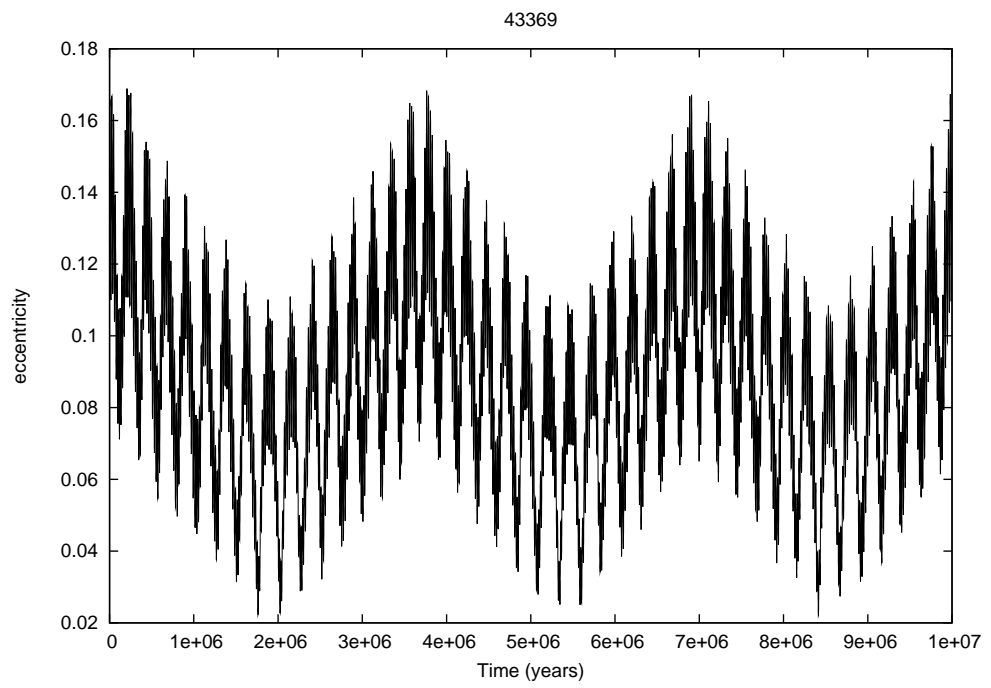

Figure 13: The mean eccentricity of (43369) $2000 \mathrm{WP}_{3}$ shows an oscillation due to the resonance $g-s-g_{5}+s_{6}$. The maxima and minima of the periodic term in eccentricity occur when the critical argument of Figure 12 passes through $0^{\circ}$. 


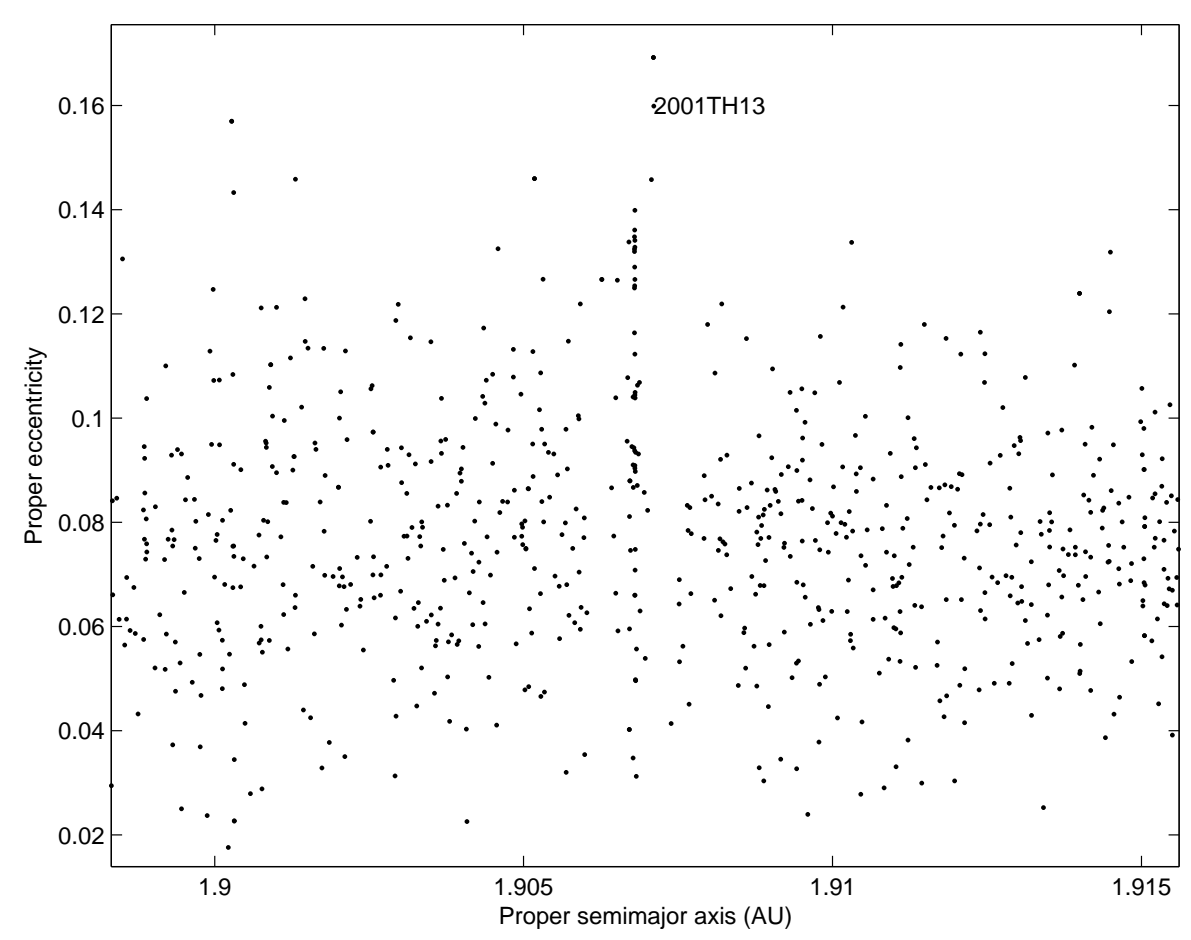

Figure 14: The proper elements $a_{p}$ and $e_{p}$ for the Hungaria with $a_{p}$ close to 1.9068 $\mathrm{AU}$, which is the location of the mean motion resonance $n / n_{4}=5 / 7$.

different mean motion resonances are involved, but the one dominating in the last $1 \mathrm{My}$ is the 5/7 resonance with Mars.

This is better seen from Figure 16, showing one critical argument of this resonance, with long episodes of libration (horizontal segments) in the last million year, preceded in the previous million years by libration in at least two other resonances, most likely three-body ones (inclined segments). This orbit is clearly chaotic, and indeed the maximum LCE is estimated at $2.8 \times 10^{-4} \mathrm{y}^{-1}$, that is the Lyapounov time is only $\simeq 3,500 \mathrm{y}$. On a time scale longer than our numerical integrations this object, and the other ones in this resonance, will have deep encounters with Mars and end up by exiting from the region. Moreover, these resonances maintain a comparatively large dispersion of proper eccentricities for all the objects in the Hungaria region, including those originally belonging to the Hungaria family. 


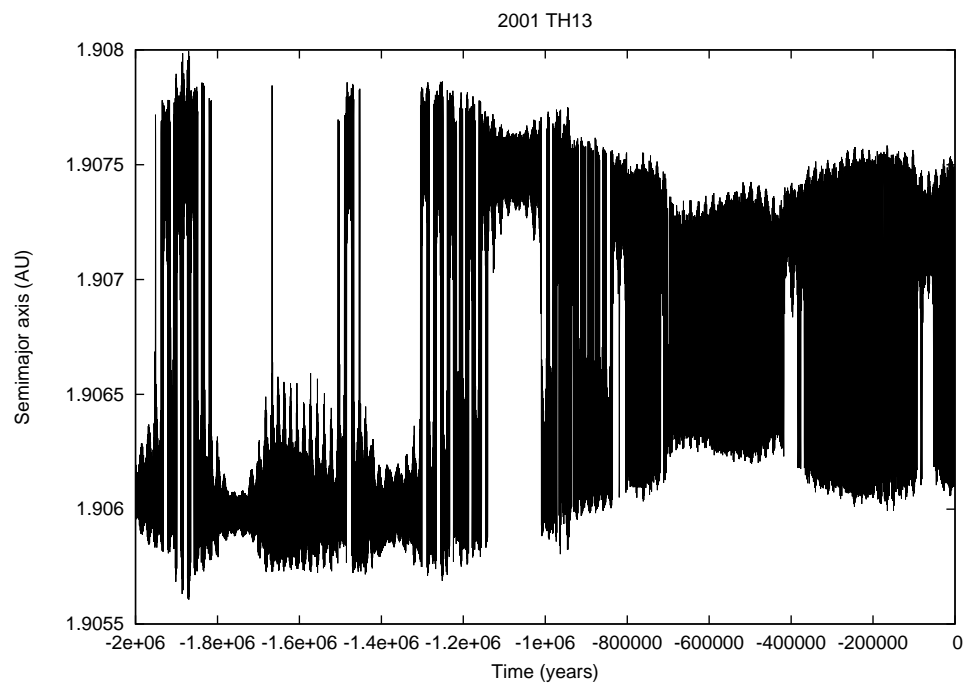

Figure 15: The semimajor axis of $2001 \mathrm{TH}_{13}$ has been digitally filtered to remove perturbations with periods up to 300 years. The largest amplitude oscillations are due to the $5 / 7$ resonance with Mars, sometimes replaced by smaller oscillations with different centers, that is in different resonances.

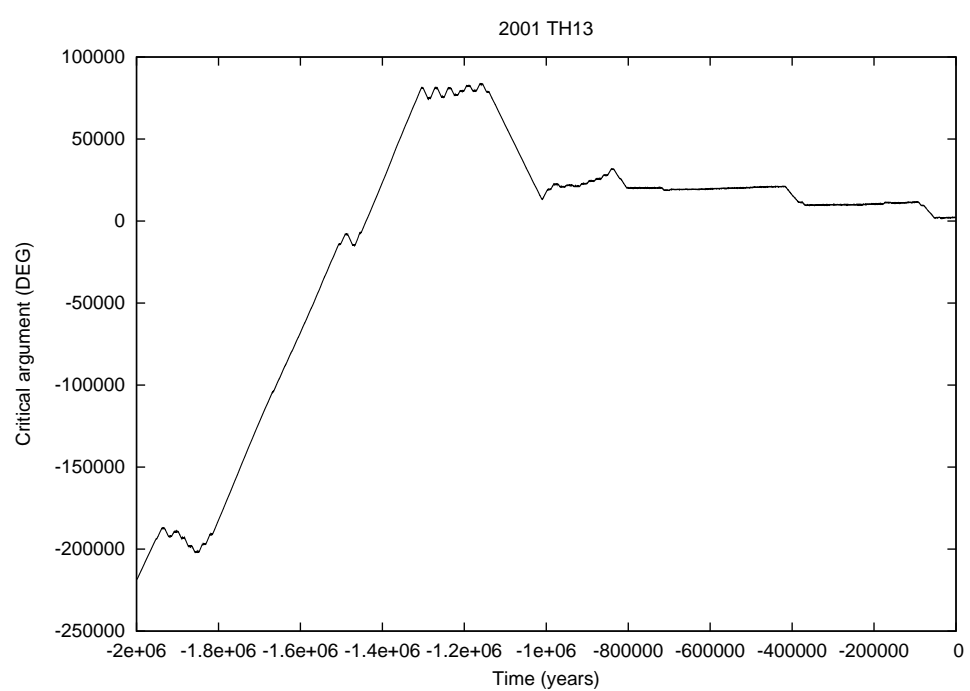

Figure 16: The critical argument $7 \lambda-5 \lambda_{4}-2 \varpi$ for asteroid $2001 \mathrm{TH}_{13}$. Alternation between libration and circulation takes place in the last million years, previously this argument is circulating, with a reversal of the circulation around 1,200,000 years ago and short episodes of libration. 


\subsection{Instability and leakage from the Hungaria region}

The question is how to count the Hungaria asteroids in an unstable orbit, possibly leading outside the region. Note that the time scales for leakage from the region must be significantly longer that the $10 \mathrm{My}$ of our longest numerical integrations: this implies we have to extrapolate from a behavior which is comparatively rare in the time span we have directly investigated. Thus we need to identify two different categories of escapers: the ones driven, e.g. by secular resonances, to orbital elements well outside of the Hungaria region, and the ones in which the eccentricity grows to Mars crossing levels, thus they will be removed, over a time scale much longer than $10 \mathrm{My}$, by perturbations resulting from close approaches [Migliorini et al. 1998].

To assess both phenomena we use the final state of the 10 My integration, which was performed for 2170 Hungaria. Note that the other cases, which were computed for only $2 \mathrm{My}$, did show a significantly better stability of proper elements and lower, in most cases insignificant, LCE, thus we can assume they are not escapers.

The final state at the end of the integration of $10 \mathrm{My}$, in terms of mean orbital elements, shows 23 asteroids with final state outside the boundaries $1.8<a<2 \mathrm{AU}, 15^{\circ}<I<29^{\circ}$ and $e<0.2$. There are additional 109 asteroids still in that region but with $q<1.65$ AU. Only some asteroids in these two groups are already experiencing close approaches to Mars, see Table 1, but a close approach to Mars is a rare event because of the high inclination of the Hungaria and also the secular evolution of the eccentricities.

The question is whether we can characterize the rate of asteroid loss from the Hungaria region, e.g., can we estimate the half life of the Hungaria? If we take 23 as the number of escapers in $10 \mathrm{My}$, out of a sample of 4424, the fraction escaping is $0.52 \%$, and assuming an exponential behavior we obtain a half life of $960 \mathrm{My}$. This number should be taken just as an order of magnitude, because the behavior is not really exponential like that of a radioactive element, still this value is very large and in disagreement with the results of the numerical experiments used by [Migliorini et al. 1998]. One difference is that this refers to a large sample, including many smaller asteroids, in a large majority belonging to the family (see next section), while [Migliorini et al. 1998] used the 56 Hungaria larger than $5 \mathrm{~km}$ in diameter known as of 1998, out of which very few are members of the Hungaria family (see the discussion in Section 4 and in particular Figure 19). Another possible source of the discrepancy is that we are using an accurate numerical integrator as opposed to an approximate symplectic integrator, which 
introduces another purely numerical source of instability.

\subsection{Non-gravitational perturbations}

All the above discussion refers to a purely conservative orbit propagation. Of course there are non-gravitational effects, mostly the Yarkovsky effect, which have not been included in the dynamical model of the numerical integrations but can make a significant difference in the long term stability.

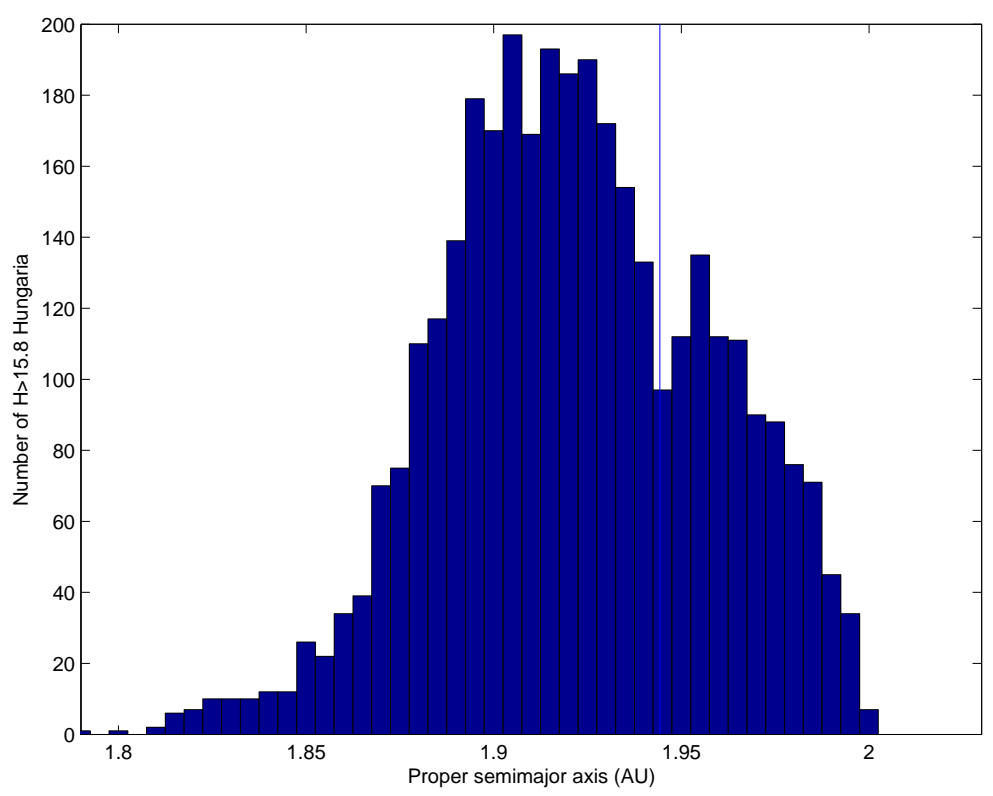

Figure 17: Histogram of the proper semimajor axis $a_{p}$ of the Hungaria asteroids. The vertical line marks the $a_{p}$ value for (434) Hungaria.

There are three main features in the $a_{p}$ histogram of Figure 17 clearly pointing to the Yarkovsky effect (and possibly other non-gravitational effects) as the main cause:

1. the spread of $a_{p}$, even after removing the lower density tail with $a_{p}<$ 1.87 , is at least an order of magnitude larger than the one implied by a reasonable ejection velocity from a parent body which could be formed with the mass present in the Hungaria region;

2 . the $a_{p}=1.9443$ value of the brightest asteroid, (434) Hungaria, corresponds to a dip in the histogram, which shows a significant bimodality; 
3. the left hump, for lower $a_{p}$, is significantly bigger than the right one, for larger $a_{p}$, with 2853 Hungaria in $1.87<a_{p}<1.9443$ and 1263 in $a_{p}>1.9443$, for a ratio 2.26 .

The total volume of all the Hungaria asteroids can be estimated as follows: the albedo of (434) Hungaria is estimated at 0.38, thus the diameter is $\sim 12.5$ $\mathrm{km}$, in agreement with [Shepard et al. 2008]. If we assume all the Hungaria asteroids to have the same albedo, then the volume of all of them together can be estimated at $\sim 15$ times the one of (434). An hypothetical parent body, even if all the Hungaria asteroids were to belong to the family, would have a diameter of $31 \mathrm{~km}$. The escape velocity from such a body would be uncertain because of the unknown density, but should not exceed $17 \mathrm{~m} / \mathrm{s}$ (corresponding to a density of $\left.3 \mathrm{~g} / \mathrm{cm}^{3}\right)$. The fact that some Hungaria asteroids have a very different (much smaller) albedo only contributes to decrease this estimate, because these objects would be background, not part of the collisional family.

Thus 1) implies that the spread has not been fixed at the origin of the family, but must have been increased by non-gravitational perturbations acting in a synergy with unstable gravitational perturbations. 2) indicates that smaller fragments have preferentially evacuated the neighborhood of (434) Hungaria, which cannot be due to gravitational instabilities, not found near that value of $a_{p}=1.9443$.

The asymmetry 3 ) is not due to the cut at $2 \mathrm{AU}$, because the two lobes are very different in numbers well before the cut. The difference in apparent magnitude as seen from Earth between 1.97 and 1.90 AU is not more than 0.16 magnitudes, thus this does not justify a difference due to observational selection more than 20 to $25 \%$.

On the contrary, it is comparatively easy to explain all these features by assuming that there is indeed a Hungaria family of asteroids which includes most (but by no means all) of the asteroids belonging to the Hungaria region ${ }^{9}$, and that such family is the result of a collisional disruption, with (434) Hungaria as the largest remnant, occurred long ago (hundreds of millions years), a time long enough for large non-gravitational perturbation.

A reasonable value for the Yarkovsky main effect, which is a secular change in the osculating semimajor axis, on a Hungaria with a diameter

\footnotetext{
${ }^{9}$ Following [Warner et al. 2009], we will use the expressions "Hungaria asteroids" and "Hungaria group" to indicate all the Hungaria, but we will explicitly say "Hungaria family asteroid" for the family members; the non-family asteroids will be called "background".
} 
of $1 \mathrm{~km}$ could be $\simeq 2.3 \times 10^{-4} \mathrm{AU} / \mathrm{My}$. This value is obtained by scaling from the best estimated value for (101955) $1999 \mathrm{RQ}_{36}$ [Milani et al. 2009], but please note that, by taking into account all the uncertainties and the missing data to constrain densities and thermal properties, this value could well be wrong by factor 2 [Vokrouhlický et al. 2000][Figure 1]. Such value is also very sensitive to the obliquity $\epsilon$ of the rotation axis for each asteroid, in a way which is well represented by a factor $\cos \epsilon$ : a positive value with a maximum at $\simeq 2.3 \times 10^{-4} \mathrm{AU} / \mathrm{My}$ could apply to a prograde rotator with $\epsilon=0^{\circ}$ obliquity, the opposite value would be the maximum rate of decrease for a retrograde rotator with $\epsilon=180^{\circ}$, with all intermediate values possible for intermediate obliquities.

Then most features of Figure 17 could result as a consequence of a preferential retrograde rotation of the Hungaria. This asymmetry could be a consequence of the YORP effect, another form of non-gravitational perturbation resulting in evolution of the asteroids spin state, although a fully self-consistent theory of the evolution of the spin state of an asteroid over hundreds of million years is not yet available. Anyway, the asymmetry in the distribution of $a_{p}$ is a fact, which becomes observable by using a large enough catalogue of proper elements, while the cause remains to be firmly established.

As for the central gap, an almost zero Yarkovsky effect is obtained only for a narrow range of obliquities around $90^{\circ}$, which tend to be depopulated by the YORP effect, thus the gap near the "origin" of the family $a_{p}$ distribution [Vokrouhlický et al. 2006a, Vokrouhlický et al. 2006b]. The origin is very near to the $a_{p}$ of (434) Hungaria but is not the "center" of the current distribution ${ }^{10}$.

To confirm these conclusions in a more quantitative way we need some additional information on the Hungaria family population and structure, thus this subject will be discussed again in the next Section.

\section{Family classification}

In order to identify possible dynamical families in the Hungaria region, we applied the hierarchical clustering method (HCM), already used in a number

\footnotetext{
${ }^{10}$ We disagree with the method used in [Warner et al. 2009] to compute the "origin" of the family $a_{p}$ distribution by using a computation of the mean value, or even a center of mass. If the perturbations are asymmetric, the center of mass is not conserved.
} 
of previous searches for families in the asteroid main belt [Zappalà et al. 1990, Zappalà et al. 1994, Zappalà et al. 1995]. The HCM is based on the idea of analyzing the proper elements space $a_{p}, e_{p}, \sin I_{p}$, looking for concentrations of objects that cannot be due to chance.

\subsection{Selection of a metric}

The first step of the analysis consists of the definition of a metric in the proper elements space, needed to quantify the mutual distances between the different objects present in the sample. As extensively discussed in the papers just mentioned above, a standard metric has been adopted in most family identification analyzes. This metric, first introduced by [Zappalà et al. 1990], is expressed in the following form:

$$
\Delta=n a_{p} \sqrt{k_{1}\left(\frac{\delta a_{p}}{a_{p}}\right)^{2}+k_{2} \delta e_{p}^{2}+k_{3} \delta I_{p}^{2}}
$$

where $\Delta$ is the distance function and the values of the coefficients are $k_{1}=$ $5 / 4, k_{2}=k_{3}=2$. According to the above definition, the distance has the dimension of a velocity (expressed in $\mathrm{m} / \mathrm{s}$ ). In family studies it is natural to express the distance between two objects in terms of the difference of velocity that must be imparted to achieve the observed difference in orbital elements, according to classical Gauss' equations, with plausible assumptions on the unknown anomaly at the time of the impact.

\subsection{Hierarchical clustering}

The introduction of a metric in the proper elements space allows us to analyze the clusters of objects present in a given volume of this space: clusters are subsets of objects such that the distance between a given member and the closest member belonging to the same cluster is below a given distance control. Different clusters are found at different values of the distance control, and it is useful to examine how the objects can be grouped in clusters, and how persistent these clusters are at different distance levels. This is done by means of procedures extensively explained in the literature, and the results are usually expressed by means of "stalactite diagrams", a graphical representation first introduced by [Zappalà et al. 1990]. This representation is effective in displaying how a given population of asteroids located in a certain region of the proper elements space tends to be distributed into a number of separate clusters and how these clusters change as a function of the assumed 


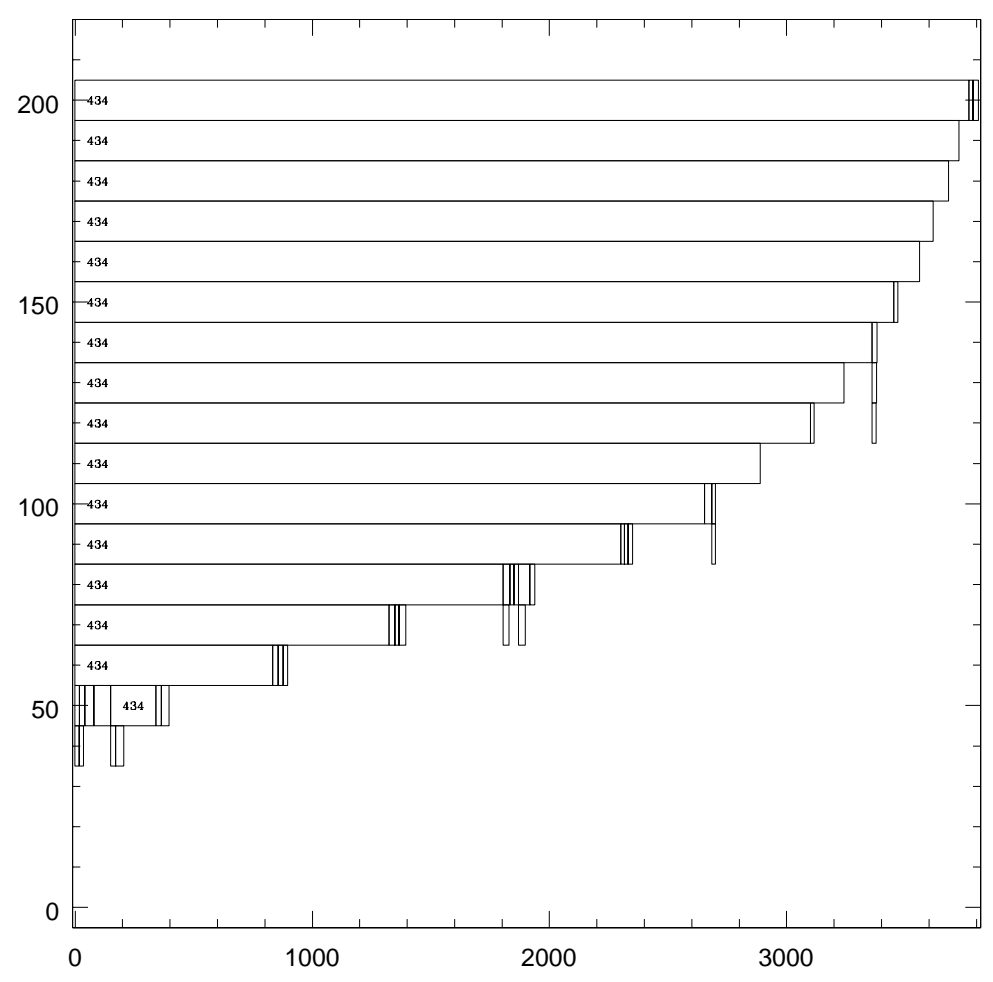

Figure 18: The stalactite diagram for our sample of Hungaria asteroids.

distance level. In these diagrams well defined families appear as deep and robust stalactite branches, whereas non-significant groupings appear as short branches quickly merging with larger groups at larger distances.

Figure 18 shows the stalactite diagram for our sample of Hungaria asteroids proper elements. In particular, the figure shows the existing groupings including at least 15 objects at different levels of distance, with steps of 10 $\mathrm{m} / \mathrm{s}$. It is easy to see that the Hungaria population seems to belong to one big and fairly compact cluster, that tends to be increasingly eroded as we go down to smaller distance levels. Small sub-groupings tend to form as we follow the main stalactite down to its lower tip, but these sub-branches are small and short. Only at the very low-end of the main stalactite there is a complete splitting of the main cluster into a handful of small and short branches, but based on a purely visual inspection of the diagram none seems 
to be very significant.

\subsection{The background problem}

In the classical application of the HCM, the statistical significance of a family, visually represented as a deep stalactite branch, was evaluated by introducing the so-called "quasi-random level", namely a critical distance level that, simply speaking, could not be attained by a random distribution of objects in a given volume of the proper elements space.

Reliable families were identified as clusters that could reach distances well below the quasi-random level, being therefore interpreted as groupings of closely packed members that cannot be produced by purely random fluctuations in the distribution of proper elements.

In turn, the quasi-random level was estimated by generating quasi-random populations of fictitious objects in a given region of the proper elements space. These quasi-random populations were generated by assigning randomly the proper elements of each object, but with the constraint that the resulting histograms in $a_{p}, e_{p}$ and $\sin I_{p}$ for the synthetic population separately fitted the corresponding histograms of the real population. In order to avoid that very compact and populous families significantly affect the proper elements histograms of the real population (thus also of the supposed random background), procedures were developed to first remove from the original population objects belonging to very evident concentrations.

The problem with the Hungaria asteroids is that the method just described to derive a quasi-random level can hardly be applied in this case, due to the fact that we are in a situation in which a single, very large, cluster dominates the whole population present in this region of the proper elements space. As a consequence, the procedure used in the past to derive a quasi-random level is not reliable, being based on a circular argument: the reliability of the family is tested with a random background, which in turn is computed by assuming the family membership. A nominal quasi-random level of $60 \mathrm{~m} / \mathrm{s}$ is found by a tentative application of the standard techniques described above, without any a priori elimination of objects to remove any large initial concentration. However, this result is affected by a large uncertainty, and can be misleading, because the supposed random background computed without removing the dominant family is significantly more dense than the "real" background we cannot identify.

In this situation, we do not introduce a quasi-random level for the population, but we just point out that the obtained stalactite diagram is typical 
of situations in which a single, overwhelming cluster dominates a given population. In other words, our HCM analysis suggests the presence of only one large family in the Hungaria region, but the boundaries of this family, and its membership, cannot be unequivocally determined.

Only some different pieces of evidence can be really decisive in constraining the problem. Among these different pieces of evidence, we mention (1) the general relation that can be found between $a_{p}, e_{p}$, sin $I_{p}$ and the absolute magnitude $H$; (2) the distribution of the colors of the Hungaria asteroids. We also note that other kind of physical information could also be taken into account, but we must face the fact that available data are scarce.

[Gil-Hutton et al. 2007] analyzed available polarimetric data for Hungaria objects, and they found heterogeneity in albedo, much more than expected if the Hungaria were to be all E-class asteroids. These findings suggest that, even if a family is present, it does not include the whole population of the Hungaria region, since families are known to be as a general rule quite homogeneous in composition [Cellino et al. 2002]. This compositionally different subgroup could be background or maybe contain a smaller separate family. However, to accept this as a rigorous conclusion we need to investigate further by using accurate and homogeneous data.

\subsection{Family structure and its time evolution}

As we have discussed in Section 3, the current distribution of proper elements of the Hungaria, even assuming we could select the exact membership, cannot reflect the distribution of original ejection velocities because the changes introduced by the combined effect of gravitational and nongravitational perturbations are by far larger.

There are few cases in which gravitational perturbations can change macroscopically the semimajor axis, and these are limited to very unstable regions where it is not possible to find a concentration of asteroids. Thus it is simpler to analyze the case of the proper semimajor axis $a_{p}$, which in the Hungaria region essentially changes only because of the Yarkovsky effect (apart from the few objects with close approaches to Mars, listed in Table 1).

All the non-gravitational perturbations depend upon the small parameter area to mass ratio, which is, for a given density, inversely proportional to the diameter $D$. If we assume all the Hungaria asteroids have the same albedo and density, the value of $D$ can be estimated for all of the objects from $H$ and the non-gravitational perturbations small parameter is proportional to $1 / D$ for all. If a Hungaria asteroid is from the background, not from the 
family, these estimates may well be incorrect, in particular the value of $1 / D$ will be smaller because the albedo could be much smaller than 0.38 .

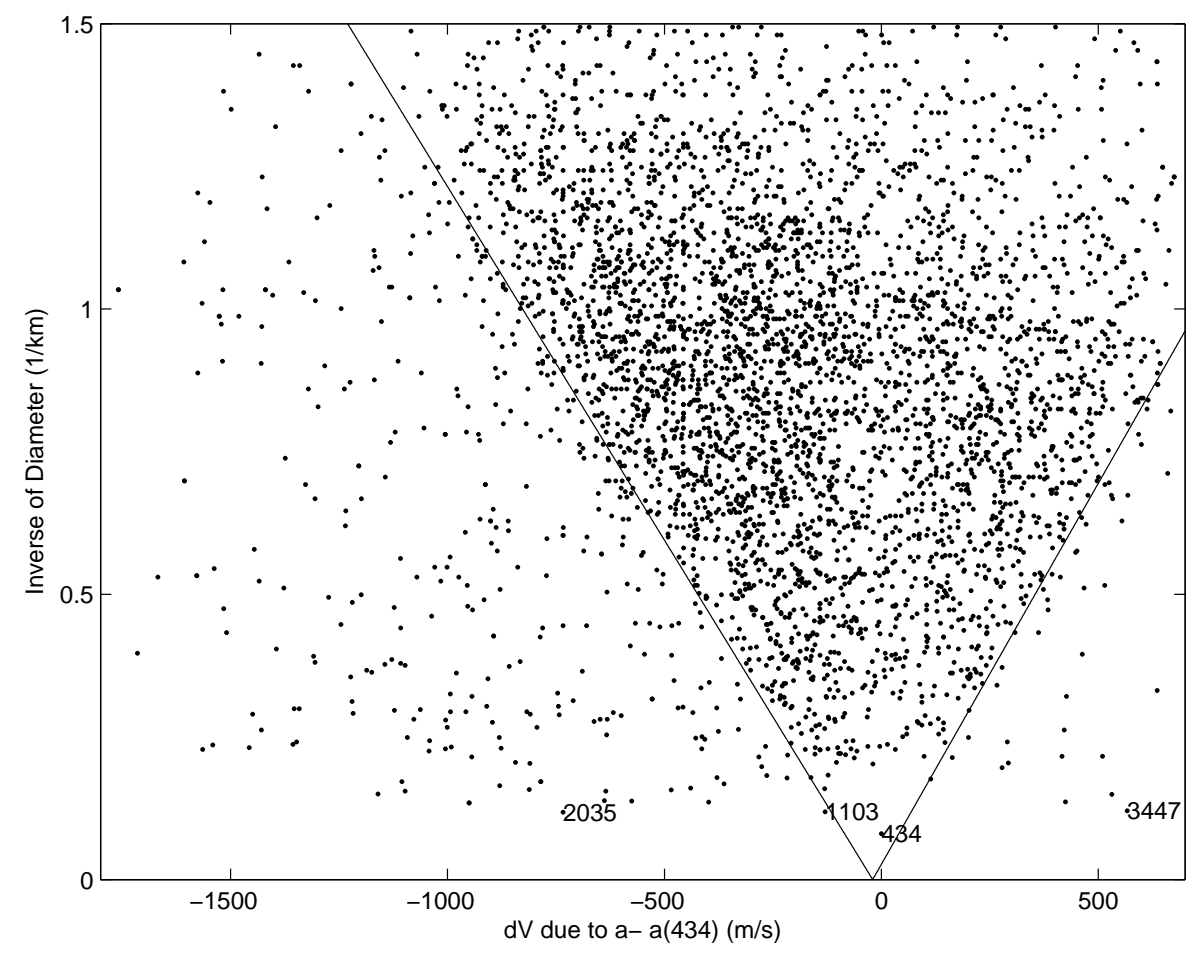

Figure 19: The velocity metric due to difference in $a_{p}$ with respect to (434) vs. the inverse diameter $1 / D$ for our sample of Hungaria asteroids, assuming the albedo is 0.38 for all.

Thus in Figure 19 we have plotted on the abscissa the velocity change corresponding to the change in $a_{p}$ with respect to (434), according to the first term under square root in metrics (1): the conversion factor is $d V \simeq$ $12179 d a_{p}$ for $d V$ in $\mathrm{m} / \mathrm{s}, d a_{p}$ in AU. On the ordinate we have the estimated $1 / D$. We have also labeled with the asteroid number the points corresponding to the largest Hungaria, with diameter $>8 \mathrm{~km}$. The straight lines forming a $\mathrm{V}$ shape have been selected visually, with the vertex of the $\mathrm{V}$ at the point with coordinates $(-20,0)$ because $20 \mathrm{~m} / \mathrm{s}$ is a rough estimate of the value of the Yarkovsky effect on (434), taking into account that its spin is prograde but with an axis near the orbital plane [Durech 2007]. As an example, an estimate for the location of the hypothetical parent body in this plot would 
be $(-20,1 / 31)$; however, $31 \mathrm{~km}$ is an upper bound, not necessarily the actual diameter. The slopes of the two lines are: for the positive $d a_{p}, d V$ increases by about $+750 \mathrm{~m} / \mathrm{s}$ for each increase of $1 / D$ by $1 \mathrm{~km}^{-1}$, for negative $d a_{p}, d V$ changes by about $-810 \mathrm{~m} / \mathrm{s}$ for the same increase of $1 / D$.

Given the simple procedure, the values of the slopes are approximate and we cannot claim to have accurately estimated the difference among the two. If the source of the $\mathrm{V}$ shape was just the distribution in $\cos \epsilon$, the two slopes should be the same, the one for larger $a_{p}$ corresponding to prograde rotators with $\epsilon=0^{\circ}$ and the one for lower $a_{p}$ for retrograde rotators with $\epsilon=180^{\circ}$. However, the average $a_{p}$ for the lobe on the left of Figure 17 is $\simeq 1.90 \mathrm{AU}$, the one for the lobe on the right is 1.96. The non-gravitational perturbations also contain as a factor the inverse square of the distance from the Sun: for this effect only, the two slopes should have a ratio $(1.90 / 1.96)^{2}=0.94$, while the ratio with our rough measuring method is 0.93 .

These slopes can also be used to weakly constrain the age of the family. For a $1 \mathrm{~km}$ diameter asteroid, the maximum prograde total change is +0.0615 $\mathrm{AU}$, the maximum retrograde change is $-0.663 \mathrm{AU}$. If we adopt the value of $2.3 \times 10^{-4} \mathrm{AU} / \mathrm{My}$ for the Yarkovsky secular perturbation on a $1 \mathrm{~km}$ Hungaria, we get values around 274 million years for the formation of the family. Given the lack of knowledge on the physical parameters controlling the Yarkovsky effect, we do not consider that these values are significantly in disagreement with the value of $500 \mathrm{My}$ given by [Warner et al. 2009].

It should be emphasized that, if no family was present in the Hungaria zone, there would be no reason to see a dense triangular domain in the plot shown in Figure 19: the plane should be expected to be rather uniformly populated, apart from a general increase of the number of objects going towards fainter $H$ values, but without any preferential pattern in terms of proper semi-major axis. Thus the distribution found in this and in the later Figures indicate that a major fraction of the Hungaria belongs to one single family, but this family does not include the whole Hungaria population.

Figure 20 also suggests that one single cluster dominates the population, but the triangular domain is barely visible and the dispersion in $e_{p}$ is large. The Yarkovsky effect is not expected to produce directly a large size-dependent spreading in eccentricity. However, the secular perturbation in semimajor axis, which is size-dependent, does result in passage through many small mean motion resonances with temporary captures in resonance which are very effective in dispersing the eccentricity. Thus the surprisingly large dispersion in $e_{p}$, which is weakly dependent upon the size, should not be 


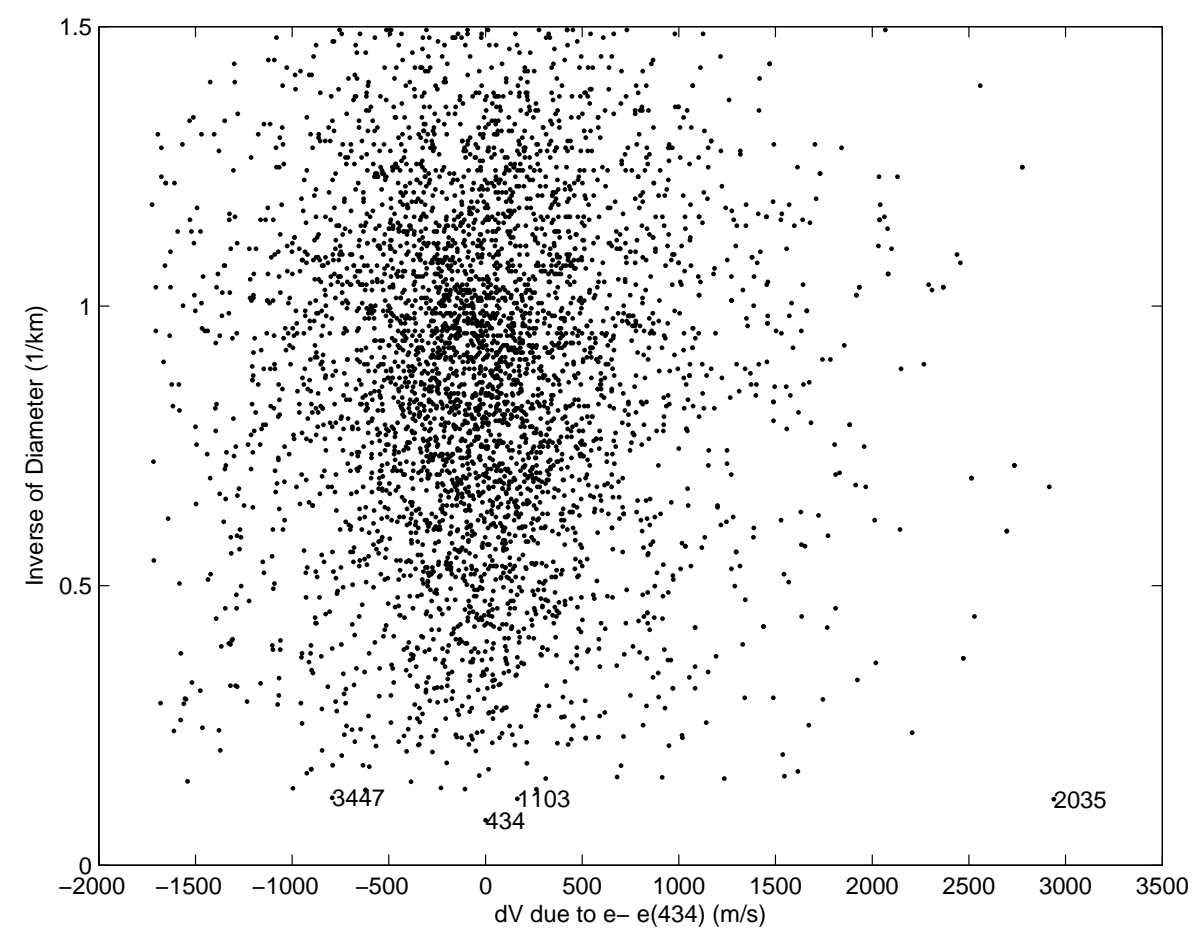

Figure 20: The velocity metric due to difference in $e_{p}$ with respect to (434) vs. the inverse diameter $1 / D$ for our sample of Hungaria asteroids.

interpreted as an indication that many asteroids belong to the background: the Hungaria with either very large of very small $e_{p}$ may well belong to the family. As the example of $2001 \mathrm{TH}_{13}$ shows (Figure 14), an asteroid may have a currently large $e_{p}$ because of a mean motion resonance, being an escaper from the family rather than an interloper in the family.

A very instructive opposite example is the asteroid (2035) Stearns, which has a large difference with respect to (434) in all three proper elements, including an extreme value of $e_{p}=0.1760$. However, by looking at an enlargement of the $\left(a_{p}, e_{p}\right)$ plot, such as Figure 14 but with $a_{p}$ close to $1.884 \mathrm{AU}$, it is possible to find evidence for a mean motion resonance (probably a 3-body one) in which several Hungaria are involved: the resonance can push up the eccentricity, and this independently from the size of the asteroid. Thus, as far as the value of $e_{p}$ (also of $\sin I_{p}$ ) is concerned, (2035) could have been, in the past, in the densest core of the Hungaria population, and still it is not a 


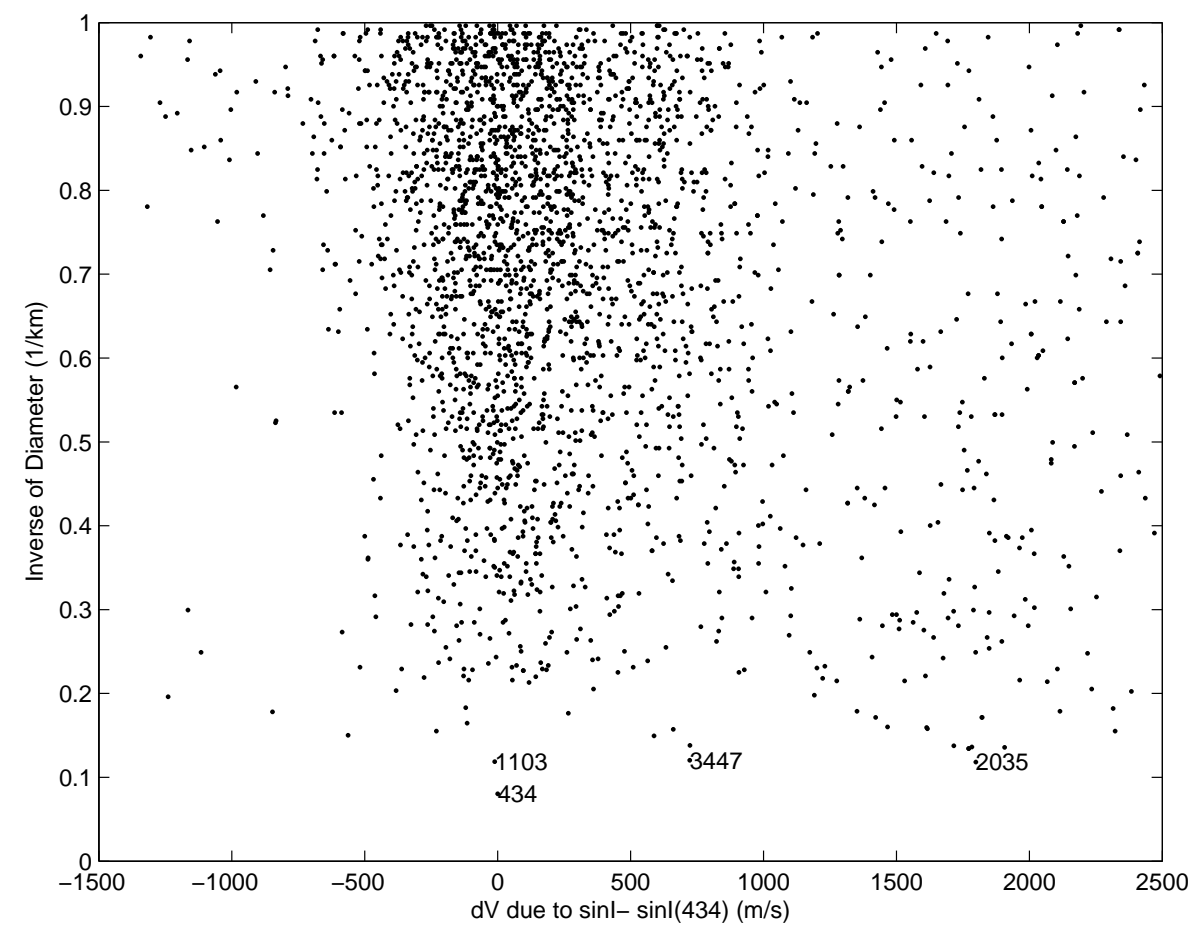

Figure 21: The velocity metric due to difference in $\sin I_{p}$ with respect to (434) vs. the inverse diameter $1 / D$ for our sample of Hungaria asteroids.

member, that is a fragment of the same parent body. The reason for considering (2035) as a background asteroid is that the difference in $a_{p}$ with (434) is by far too large for an object of that size, as it is clear from Figure 19. That is, an asteroid can be excluded from the family because of its position in the four-dimensional space $\left(a_{p}, e_{p}, \sin I_{p}, H\right)$ rather than by the proper elements alone. Another example of this is (3447) Burchalter, which has colors consistent with the same composition as (434) [Carvano et al. 2001], and still is not a fragment of the same parent body.

In Figure 21 some narrower but not negligible triangular domain can be seen; still this could not be a trace of an original size-ejection velocity relation, but has been sharply modified by subsequent dynamical evolution. In the case of the proper inclination the changes due directly to the Yarkovsky effect should be negligible. However, mean motion resonances also affect the inclination, although somewhat less than the eccentricity. The secular reso- 
nances containing the $s$ frequency can act in collaboration with Yarkovsky to generate changes in inclination, which the two effects separately could not produce: see a very good example in [Warner et al. 2009][Figure 8].

Still, our dynamical and family arguments above do not completely explain the distribution of $\sin I_{p}$, because there appears to be a grouping separate from the main family for $\sin I_{p}>0.4$, see also Figure 8 . It is possible to confirm, by a plot like Figure 19 limited to the Hungaria with $I_{p}>23.5^{\circ}$, that this grouping is not centered around the origin of the Hungaria family (at $-20 \mathrm{~m} / \mathrm{s}$ ) but much on the left (around $-600 \mathrm{~m} / \mathrm{s}$ ). This could later, when much more data are available, turn out to be a separate family, but then it would be significantly older than the Hungaria family. The reason why such family was not suggested by the HCM method is the well known problem of "chaining": there are Hungaria family members spilling out of the lower $I_{p}$ region because of mean motion resonances which form bridges to the higher $I_{p}$ grouping. One reason why we cannot obtain a reliable identification of such a second family is that there is an observational bias against discovery of high inclination Hungaria.

\subsection{Color information and family membership}

An analysis of the available SDSS colors for the asteroids of our sample can also be useful to infer some information on the existence and membership of one big dynamical family in the Hungaria region. For this purpose we used the Principal Component Analysis (hereafter PCA), which obtains, by a linear transformation, from a number of correlated variables a smaller number of uncorrelated variables, called principal components. The first principal component accounts for as much of the variability in the data as possible, and each succeeding orthogonal component accounts for as much of the remaining variability as possible. In this application, the PCA creates two linear combinations of the five SDSS colors that maximize the separation between the taxonomic types. In order to calculate the first two principal components we adopted the following relationships from [Nesvorný et al. 2005]:

$$
\begin{aligned}
& P C_{1}=0.396(u-g)+0.553(g-r)+0.567(g-i)+0.465(g-z), \\
& P C_{2}=-0.819(u-g)+0.017(g-r)+0.09(g-i)+0.567(g-z),
\end{aligned}
$$

where $u, g, r, i, z$ are the measured fluxes in five bands after correction for solar colors; for the values of solar colors see [Ivezić et al. 2001]. 


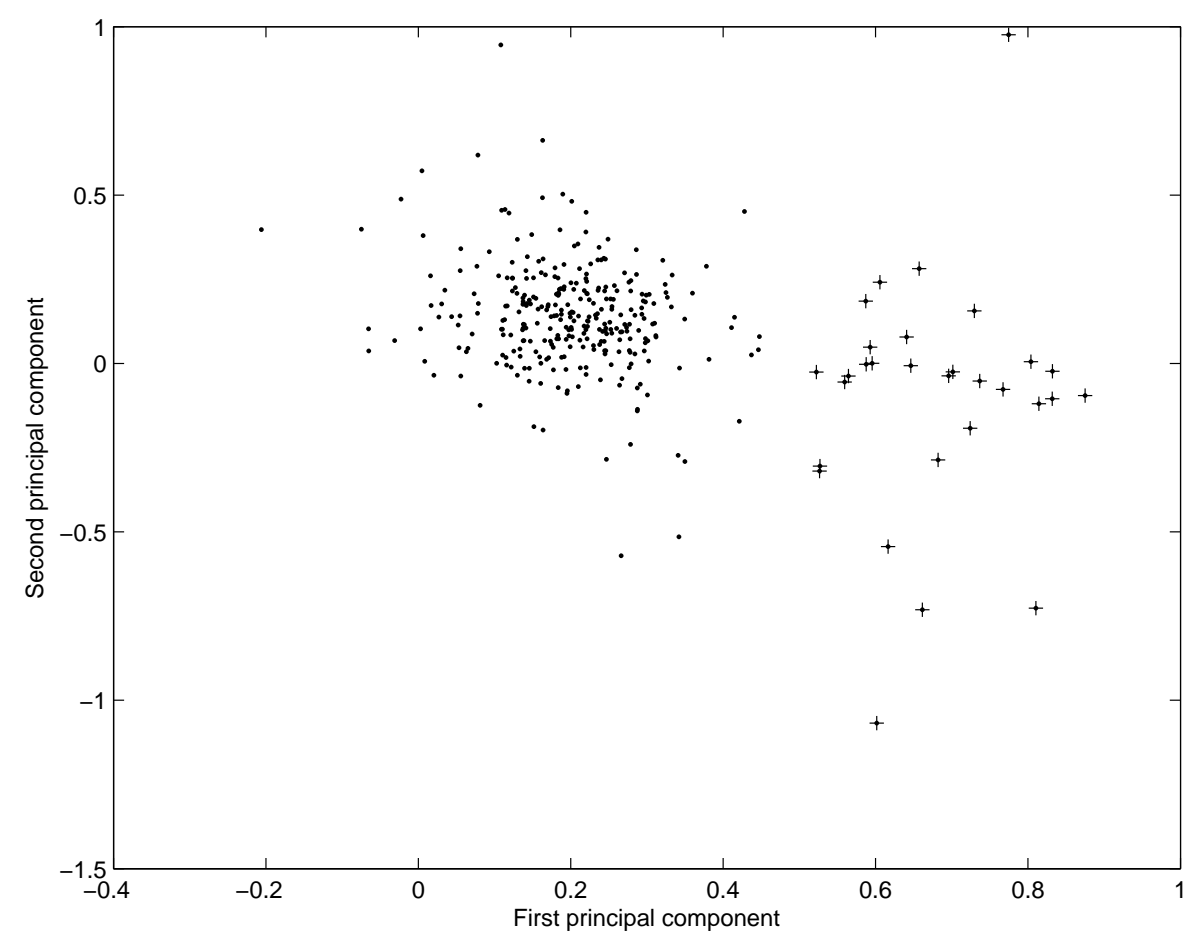

Figure 22: The Hungaria asteroids with SDSS color data plotted in the plane of the first two principal components, given by eqs. (2) and (3). The crosses mark the asteroids with clearly different spectral properties with respect to the majority of the Hungaria: these can be reliably considered as background asteroids.

We have used as source of data the third release of the SDSS MOC catalog of moving objects ${ }^{11}$, containing data for 43424 moving objects identified with known asteroids; of these 338 are Hungaria. We have not used the new release 4 of the same catalog (containing data for 104449 known asteroids) because it includes non-photometric nights, and to handle such inhomogeneous quality we would need to develop a more complicated algorithm taking into account the photometric uncertainty for each individual object.

Having computed the two first principal components of the measured colors of 338 Hungaria asteroids, plotted in Figure 22, we need to find a way to use them to obtain reliable criteria for discriminating family and background

\footnotetext{
${ }^{11}$ http://www. astro.washington. edu/users/ivezic/sdssmoc/sdssmoc3.html
} 
Hungaria. To use an existing taxonomy, among the many ones built from multicolor photometry data, which is in itself uncertain and even controversial, would not help in giving a non-controversial membership of the Hungaria family. As the most relevant example of this problem, many Hungaria can be classified as either $\mathrm{X}$ or $\mathrm{Xe}$ taxonomic class based on visible and nearinfrared photometry, but the fact is, an $\mathrm{X} / \mathrm{Xe}$ asteroid could be either an $\mathrm{E}$ asteroid, like (434), or a compositionally very different $\mathrm{C}$ asteroid, depending upon the value of the albedo. Only a source of information independent from multicolor photometry, such as far infrared observations, polarimetry, radar observations and occultations, could provide albedo and thus unambiguous taxonomy, but such additional data are available for too few Hungaria.

[Nesvorný et al. 2005] propose a method for classification into taxonomic complexes such as $S, C$ and $X$ based on the SDSS principal components: by using the same method we classified the 338 Hungaria asteroids, which are in the third release of SDSS MOC, into three taxonomic complexes. We found that, according to this criterion, most of the Hungaria asteroids, $\sim 84 \%$, belong to the $X$-type, while $\sim 12 \%$ belong to the $S$-type and only $\sim 4 \%$ belong to the $C$-type. Even assuming this taxonomy of the Hungaria was as good as it can be with multicolor photometry only, still it can be used only in a statistical sense, e.g., to reject as background an asteroid classified as $\mathrm{C}$ is likely to be correct but cannot be considered sure.

The first conclusion we can draw from the above discussion is that we need to use the principal components as raw data, without the intermediary of a taxonomy. The second conclusion is that it is possible to exclude some objects from the family, that is to list them as background, with good confidence, but it is generally not possible to prove that a given Hungaria is a family member: this because either the color data may be missing, as it is still the case for most Hungaria, or they may exist but give ambiguous information on the composition similarity to (434).

Our method to generate a reliable list of background Hungaria is illustrated by Figure 23 and is the following: we combine information from Figure 19, by discarding the objects lying well outside the $\mathrm{V}$ shape marked there, and from Figure 22, by selecting an arbitrary but safe region, such as $P C_{1}>0.5$, containing objects which could not possibly have the same composition as (434), and thus have to be background. The union of the two lists of objects discarded for these two reasons is our "safe background list"; of course some asteroids may be considered background for both reasons. On the contrary we are not selecting by the value of the difference in either $e_{p}$ 


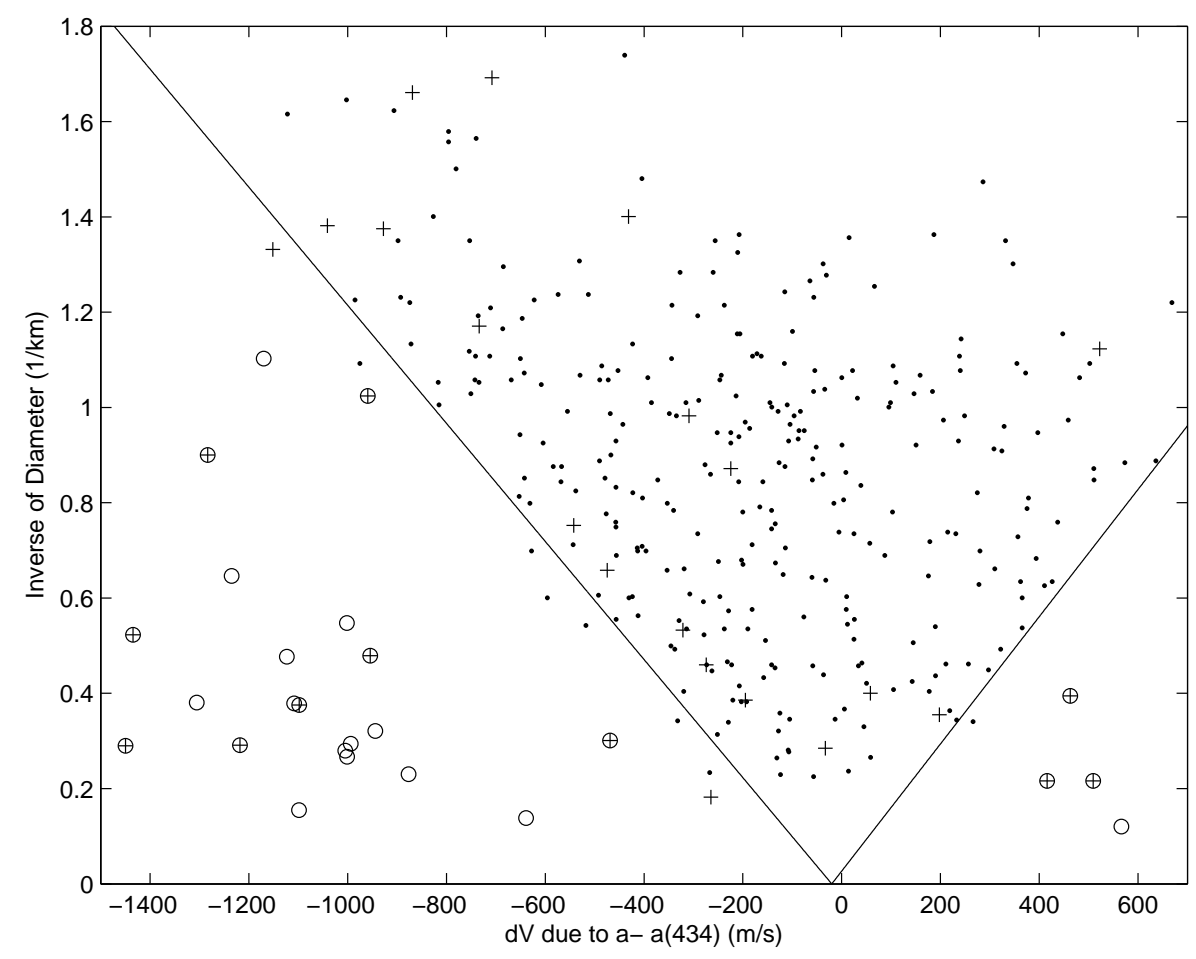

Figure 23: The Hungaria asteroids with SDSS color data plotted in the plane of $1 / D$ and $d V$ due to difference in $a_{p}$ with (434). The crosses mark the asteroids with a clearly different composition with respect to the majority, which can be reliably considered as background asteroids. The $\mathrm{V}$ shaped boundary is the same of Figure 19, and the circles mark the asteroids which do not belong to the family because the Yarkovsky effect is not enough to reach their value of $a_{p}$.

or $\sin I_{p}$, because large values of these do not exclude that the object was originally inside the dense core of the family long time ago (as discussed in the previous subsection), and therefore could in fact be fragment from the same parent body, if there is no color information to contradict this possibility. The criterion we have used for color-based exclusion may be slightly improved, but the separation from the cluster visible in Figure 22 containing most Hungaria must be kept large enough for reliability.

In Table 3 we list the 44 Hungaria asteroids, for which the color data are available from SDSS MOC version 3, which we exclude from the Hungaria family and consider background. "col" indicates exclusion by SDSS colors 
Table 3: List of Hungaria asteroids not belonging to the Hungaria family, according to either one or the other of the criteria described in the text.

\begin{tabular}{|c|c|c|c|c|c|c|c|}
\hline name & $\mathrm{H}$ & $a_{p}$ & $e_{p}$ & $\sin I_{p}$ & $P C_{1}$ & $P C_{2}$ & why \\
\hline 1727 & 12.62 & 1.8541139 & $\begin{array}{r}P \\
0.0883008\end{array}$ & $\begin{array}{r}P \\
0.3485485\end{array}$ & 0.34265 & -0.01382 & $\mathrm{da}$ \\
\hline 2049 & 14.68 & 1.9490889 & 0.0737544 & 0.4105409 & 0.55949 & -0.05516 & $\mathrm{col}$ \\
\hline 3169 & 12.37 & 1.8918438 & 0.0702169 & 0.3803682 & 0.41476 & 0.13721 & da \\
\hline 3447 & 12.07 & 1.9908063 & 0.0514424 & 0.3803603 & 0.26326 & 0.03458 & da \\
\hline 4116 & 13.48 & 1.8723042 & 0.1017214 & 0.3963218 & 0.43722 & 0.02511 & da \\
\hline 4483 & 12.97 & 1.9225667 & 0.0850299 & 0.4335313 & 0.76733 & -0.07709 & col \\
\hline 4531 & 13.80 & 1.8621129 & 0.0576858 & 0.4109745 & 0.22201 & 0.12680 & da \\
\hline 5577 & 13.99 & 1.8442805 & 0.0696235 & 0.3409321 & 0.87481 & -0.09538 & col da \\
\hline 5871 & 13.90 & 1.8617944 & 0.0496582 & 0.3817541 & 0.25235 & 0.19156 & $\mathrm{da}$ \\
\hline 7579 & 13.34 & 1.9784511 & 0.0750400 & 0.3525987 & 0.59298 & 0.04869 & col da \\
\hline 8825 & 14.20 & 1.8667877 & 0.0347522 & 0.3899147 & 0.29647 & 0.03701 & $\mathrm{da}$ \\
\hline 9165 & 13.34 & 1.9861035 & 0.0921595 & 0.4217867 & 0.56441 & -0.03728 & col da \\
\hline 11437 & 14.01 & 1.8627138 & 0.0710539 & 0.4063193 & 0.28421 & 0.02839 & da \\
\hline 13111 & 13.94 & 1.9415789 & 0.1056913 & 0.4084060 & 0.70134 & -0.02481 & col \\
\hline 17483 & 14.56 & 1.8532949 & 0.0477032 & 0.3770751 & 0.42111 & -0.17139 & $\mathrm{da}$ \\
\hline 17681 & 13.98 & 1.8252601 & 0.0217839 & 0.3843822 & 0.52236 & -0.02562 & col da \\
\hline 23615 & 14.42 & 1.9605582 & 0.1140930 & 0.3890103 & 0.68209 & -0.28660 & col \\
\hline 24457 & 14.60 & 1.9283138 & 0.0637816 & 0.3937007 & 0.58767 & -0.00255 & col \\
\hline 26916 & 14.06 & 1.9057933 & 0.0749970 & 0.4281887 & 0.69579 & -0.03660 & col da \\
\hline 37635 & 14.54 & 1.8541307 & 0.0654563 & 0.3610778 & 0.83217 & -0.02332 & col da \\
\hline 43369 & 14.98 & 1.9217882 & 0.0863188 & 0.4344706 & 0.73664 & -0.05201 & col \\
\hline 51371 & 14.57 & 1.8370908 & 0.1044790 & 0.3611307 & 0.19765 & 0.08240 & $\mathrm{da}$ \\
\hline 52384 & 15.06 & 1.8520716 & 0.0966855 & 0.4166184 & 0.26426 & 0.07052 & $\mathrm{da}$ \\
\hline 53424 & 15.07 & 1.8659568 & 0.0469200 & 0.4257507 & 0.83192 & -0.10469 & col da \\
\hline 53440 & 15.30 & 1.9179153 & 0.0848201 & 0.4195656 & 0.81436 & -0.11938 & col \\
\hline 56338 & 15.26 & 1.8265179 & 0.0396548 & 0.3283429 & 0.64074 & 0.07884 & col da \\
\hline 63605 & 15.76 & 1.9053245 & 0.0800658 & 0.4292725 & 0.64624 & -0.00637 & col \\
\hline 66150 & 15.36 & 1.8620665 & 0.0837066 & 0.4068047 & 0.29307 & 0.16521 & da \\
\hline 82074 & 14.65 & 1.9822855 & 0.0338654 & 0.3715686 & 0.80387 & 0.00553 & col da \\
\hline 83990 & 15.72 & 1.8429160 & 0.0844184 & 0.3740273 & 0.20321 & 0.15136 & $\mathrm{da}$ \\
\hline 85095 & 16.37 & 1.9258867 & 0.0691143 & 0.3562747 & 0.61645 & -0.54397 & col \\
\hline 2001BX61 & 16.63 & 1.9189363 & 0.0634420 & 0.4178222 & 0.72418 & -0.19219 & col \\
\hline 2001RR46 & 16.72 & 1.8655251 & 0.0531439 & 0.4136012 & 0.58726 & 0.18497 & col da \\
\hline 2001SM68 & 17.01 & 1.8840444 & 0.0724554 & 0.3741516 & 0.60152 & -1.06757 & $\mathrm{col}$ \\
\hline 2001TP16 & 16.92 & 1.9871935 & 0.0864623 & 0.3391498 & 0.77451 & 0.97591 & col \\
\hline 2001UN66 & 16.05 & 1.8997662 & 0.0369075 & 0.3992902 & 0.60575 & 0.24145 & col \\
\hline 2002CS289 & 17.81 & 1.8861807 & 0.0694722 & 0.4383592 & 0.81029 & -0.72640 & col \\
\hline 2002PZ141 & 17.36 & 1.8681489 & 0.0512508 & 0.3684501 & 0.52643 & -0.31946 & col \\
\hline 2002QM6 & 16.88 & 1.8482364 & 0.0668657 & 0.3610398 & 0.18678 & 0.13008 & da \\
\hline 2003BG52 & 17.29 & 1.8497485 & 0.0267820 & 0.4023059 & 0.72965 & 0.15637 & col \\
\hline 2003QJ73 & 17.40 & 1.9088879 & 0.0303655 & 0.4014184 & 0.52701 & -0.30475 & col \\
\hline 2004BM111 & 16.44 & 1.8389145 & 0.0223020 & 0.3525737 & 0.59540 & 0.00043 & col da \\
\hline 2004JA13 & 17.37 & 1.8588182 & 0.0762500 & 0.3978405 & 0.66143 & -0.73109 & col \\
\hline 2004PM42 & 17.77 & 1.8729659 & 0.0901085 & 0.4199964 & 0.65725 & 0.28136 & col \\
\hline
\end{tabular}


(corresponding to crosses in Figure 23), "da" indicates exclusion by value of $a_{p}$ and $H$ incompatible with Yarkovsky evolution from the neighborhood of (434) (corresponding to circles in the figure); of course some objects are discarded for both motivations, they are marked with a crossed circle. Note that we cannot use the ratio 44/338 as an indication of the fraction representing the background in the Hungaria group, because SDSS, like every survey, has a magnitude-dependent observational bias. It is clear that the family asteroids are dominating at the smaller sizes, because of the different size distribution estimated by [Warner et al. 2009].

Looking at the distribution of the proper elements for the Hungaria which are not to be considered family members because of their SDSS colors, there is a significant fraction with $I_{p}$ above the dense core of the group, including 6 objects with $I_{p}>23.5^{\circ}$ (out of 11 for which there are SDSS colors available). Because of the small number statistics, it is difficult to reach a firm conclusion, but we can say that if there is another family including the possible grouping at high $I_{p}$, then it is likely to be of very different composition.

\section{Very close couples}

The existence of couples of asteroids with very close orbital elements has been reported many times, see [Pravec and Vokrouhlický 2009] and references therein; however, it is difficult to discriminate the really significant cases from couples which are close by chance, taking into account that the distribution of the asteroids in the orbital elements space is by no means random ${ }^{12}$.

Thanks to the set of proper elements we have computed, it is now possible to use a simple metrics in the space of the proper elements $\left(a_{p}, e_{p}, \sin I_{p}\right)$ as a first filter to select the interesting cases. In principle, the same algorithm could be applied to osculating orbital elements: however, two asteroids on significantly different orbits could happen to have very close $(a, e, \sin I)$ at some epoch, but with very different angular variables $(\Omega, \omega, \ell)$, and then the planetary perturbations would increase the distance in a comparatively short time, while similarity in proper elements is stable over long times.

Of course, since there are only three proper elements, $a_{p}, e_{p}$, sin $I_{p}$, finding a couple passing the first filter must be confirmed by a second filter, checking

\footnotetext{
${ }^{12}$ As a consequence, statistical likelihood estimates based on simplistic assumptions (e.g., Poisson distribution) are wrong.
} 
the presence of close values of the variables $(\Omega, \omega)$ at some time in the past. The couples proposed by the second filter need to be tested by a third filter, detecting the existence of some epoch in the past in which all six osculating orbital elements were very close, implying the two asteroids were close in the physical 3-dimensional space and with a low relative velocity.

When the catalogues contain many sets of proper elements, as it is the case for our catalog of 4424 proper elements sets for Hungaria ${ }^{13}$, it is useful to apply an efficient algorithm, with computational complexity of the order of $N \log N$ for $N$ sets of proper elements, to find the couples with distances lower than some control. This is obtained by first sorting by one of the proper elements (we have used $\sin I_{p}$ ) by an efficient sorting algorithm, like bubble sorting. Then the search of the nearest neighbor can be performed by a a binary search in the sorted list, followed by a sequence of tests on the other two elements; in this way the overall procedure can be very fast, if properly programmed.

\subsection{Selection by successive filtering}

Table 4 lists the couples with a distance between proper elements sets $d<4.2 \times 10^{-4}$. We have used a distance proportional to the one we have used in the family identification, namely

$$
d=\sqrt{k_{1}\left(\frac{\delta a_{p}}{a_{p}}\right)^{2}+k_{2} \delta e_{p}^{2}+k_{3} \delta I_{p}^{2}} ; k_{1}=5 / 4, k_{2}=2, k_{3}=2 .
$$

The factor $n a_{p}$ we have omitted here is about $20 \mathrm{~km} / \mathrm{s}$, thus the distance limit we have set corresponds to less than $9 \mathrm{~m} / \mathrm{s}$, a value by far lower than what is generally used in family classification. Still, we have found 13 couples satisfying this first filter. The question is how accurate is this first filter, which is obtained in a fraction of a second of CPU time, in predicting which couples of asteroids are physically related.

The most striking case involves the numbered asteroid (88259) $2001 \mathrm{HJ}_{7}$ and the multi-opposition $1999 \mathrm{VA}_{117}$. The synthetic proper elements have extraordinarily close values, with difference in $a_{p}$ of $2.2 \times 10^{-5} \mathrm{AU}$ and of the order of $10^{-6}$ in $e_{p}, \sin I_{p}$. Currently the two objects are spaced essentially along track, with a difference in mean longitude of $\simeq 57^{\circ}$. Also the

\footnotetext{
${ }^{13}$ It is even more the case for our catalog of 209558 proper elements sets for numbered main belt asteroids, available from the AstDyS site.
} 
Table 4: Very close couples among the Hungaria asteroids, selected after filter 1: the distance $d$ is from eq. (4).

\begin{tabular}{rllrrrr}
\hline no. & name & name & $d$ & $\delta a_{p} / a_{p}$ & $\delta e_{p}$ & $\delta \sin I_{p}$ \\
\hline 1 & 88259 & 1999VA117 & 0.0000144 & +0.0000113 & -0.0000007 & +0.0000011 \\
2 & 63440 & 2004TV14 & 0.0000313 & +0.0000013 & +0.0000129 & +0.0000088 \\
3 & 92336 & 143662 & 0.0001183 & +0.0000839 & +0.0000185 & -0.0000203 \\
4 & 23998 & 2001BV47 & 0.0001501 & +0.0001190 & +0.0000005 & -0.0000099 \\
5 & 160270 & 2005UP6 & 0.0001959 & +0.0000833 & +0.0000590 & -0.0000583 \\
6 & 84203 & 2000SS4 & 0.0002075 & +0.0000881 & -0.0000122 & +0.0000871 \\
7 & 133936 & 2006QS137 & 0.0002316 & +0.0000740 & -0.0001048 & -0.0000169 \\
8 & 2002 SF64 & 2007AQ6 & 0.0002446 & -0.0001329 & +0.0000879 & -0.0000182 \\
9 & 173389 & 2002KW8 & 0.0003029 & +0.0001382 & +0.0001146 & +0.0000483 \\
10 & 27298 & 58107 & 0.0003204 & -0.0001992 & +0.0000071 & -0.0001006 \\
11 & 115216 & 166913 & 0.0003301 & +0.0002278 & +0.0000668 & -0.0000501 \\
12 & 45878 & $2001 \mathrm{CH} 35$ & 0.0003482 & -0.0002590 & +0.0000591 & -0.0000250 \\
13 & 25884 & 48527 & 0.0004012 & +0.0000761 & -0.0001089 & +0.0001616 \\
\hline
\end{tabular}

second couple, formed by (63440) and $2004 \mathrm{TV}_{14}$, is extraordinarily close: the difference in proper semimajor axis is comparable to the RMS of the osculating semimajor axis of $2004 \mathrm{TV}_{14}$. We have compared these distances with the ones we can find in a much larger sample, including proper elements for Hungaria and for numbered main belt asteroid: the couple with (88259) still is the closest, the one with (63440) is the sixth closest.

As a second filter, we have used the D-criterion [Drummond 2000], a metric measuring the similarity of 5 out of 6 Keplerian orbital elements (excluding the anomaly). Table 5 shows the results of a search, for each couple, of very low values $\left(<10^{-4}\right)$ of the $\mathrm{D}$-criterion value at some epoch in the last $2 \mathrm{My}$. The date of this event is reported for the nominal value of the initial conditions of both asteroids in the couple. Thus the couples $1,3,4,5,6,8$, and 13 have had very similar orbits at some time in a comparatively recent past, while couples $7,9,10,11$, and 12 did not.

We have also used a set of clones for each orbit of the asteroids in the couples, by assigning at random initial conditions within the current orbit uncertainty (as expressed by the covariance matrix, also available from AstDyS). In this way we have been able to check that the conclusions on the existence of an epoch of very close orbit similarity in the last $2 \mathrm{My}$ is robust with respect to the uncertainty of the orbit: this with only one exception.

The couple 2, with (63440) and $2004 \mathrm{TV}_{14}$, is an exceptional case because their elements $\Omega, \omega$ are already very close, thus the D-criterion gives an enor- 
Table 5: Very close couples selected after filter 2: couples with the nearest times in the past of close orbit similarities, obtained by the $D$-criterion. TCA1 is the time of maximum orbit similarity for the two nominal asteroid orbits; TCA2 is the mean and range of uncertainty of the same times of similarity obtained with clones. $H_{1}, H_{2}$ are the absolute magnitudes.

\begin{tabular}{cccccccc}
\hline $\mathrm{n}$ & name1 & $H_{1}$ & name2 & $H_{2}$ & $d H$ & TCA 1 [yr] & TCA 2 [yr] \\
\hline 1 & 88259 & 14.82 & $1999 \mathrm{VA} 117$ & 16.99 & 2.17 & -32000 & $-32588 \pm 687$ \\
2 & 63440 & 14.89 & $2004 \mathrm{TV} 14$ & 17.25 & 2.34 & too many & \\
3 & 92336 & 15.29 & 143662 & 16.40 & 1.11 & -348850 & $-348964 \pm 446$ \\
4 & 23998 & 15.29 & $2001 \mathrm{BV} 47$ & 16.47 & 1.18 & -406250 & $-406565 \pm 887$ \\
5 & 160270 & 16.44 & $2005 \mathrm{UP} 6$ & 17.37 & 0.93 & -1734250 & $-1646315 \pm 163035$ \\
6 & 84203 & 15.58 & $2000 \mathrm{SS} 4$ & 16.59 & 1.01 & -119159 & $-117593 \pm 4920$ \\
7 & 133936 & 16.10 & $2006 \mathrm{QS} 137$ & 16.60 & 0.50 & & \\
8 & $2002 \mathrm{SF} 64$ & 18.41 & $2007 \mathrm{AQ} 6$ & 17.39 & 1.02 & -108950 & $-113396 \pm 12938$ \\
9 & 173389 & 16.84 & $2002 \mathrm{KW} 8$ & 16.99 & 0.15 & & \\
10 & 27298 & 15.16 & 58107 & 15.49 & 0.33 & & \\
11 & 115216 & 15.70 & 166913 & 16.46 & 0.76 & & \\
12 & 45878 & 14.29 & $2001 \mathrm{CH} 35$ & 15.91 & 1.62 & & \\
13 & 25884 & 14.26 & 48527 & 15.75 & 1.49 & -422100 & $-422733 \pm 900$ \\
\hline
\end{tabular}

mous number of possible epochs for a close approach. Thus couple 2 passes the second filter, that is a low velocity close approach is possible but we get no indication on the epoch.

One suggestive feature in Table 5 is the following: the couples with close orbit similarity occurring in the last 1 My have a difference in absolute magnitude $H$ above 1 magnitude. Couple 5 , with $\Delta H=0.93$, has an orbit similarity but significantly earlier than the others. All the couples with $\Delta H<0.9$ magnitudes do not pass filter 2 (but couple 12 with $\Delta H=1.62$ is in the same category). This is not strong evidence because of the small number statistics, but there is some indication that couples which have been very close in some comparatively recent past tend to have a major and a minor partner, with mass ratio $>4$.

\subsection{Search for low velocity close encounters}

The method we have used to find the occurrence of an actual low velocity close approach in the last $2 \mathrm{My}$, with the value of the minimum distance and relative velocity at the closest approach, is labor-intensive and we would very much like to develop a more automated procedure; anyway what we have done is enough for such a small sample of couples. Having converted the output of a numerical integration for $-2 \mathrm{My}$ in Cartesian coordinates, of 
course with no digital filtering, we find the dates of minimum distance. Then we perform a shorter numerical integration, with higher sampling frequency for the output, around these dates; if necessary we iterate until the closest approach can be computed with enough accuracy.

Table 6 shows the results of such procedure for the nominal orbits of all the asteroids involved. The results of this table are especially interesting for couples 1,3 , and 13 ; for couples $4,5,6$, and 8 it is likely that a closer approach, and with lower relative velocity, could be found by either selecting some clones or by taking into account the Yarkovsky effect.

Table 6: Very close couples selected after filter 3: couples with the times in the past of a low relative velocity close approach; minimum distance and relative velocity are given.

\begin{tabular}{rccrrrr}
\hline $\mathrm{n}$ & name1 & name2 & $\begin{array}{r}d H \\
{[\mathrm{mag}]}\end{array}$ & $\begin{array}{r}\text { epoch } \\
{[\mathrm{yr}]}\end{array}$ & $\begin{array}{r}\text { min. dist. } \\
{[\mathrm{km}]}\end{array}$ & $\begin{array}{r}\text { rel. vel. } \\
{[\mathrm{m} / \mathrm{s}]}\end{array}$ \\
\hline 1 & 88259 & 1999 VA117 & 2.17 & -32453 & 2633 & 0.12 \\
2 & 63440 & $2004 \mathrm{TV} 14$ & 2.34 & -908100 & 111099 & 2.60 \\
3 & 92336 & 143662 & 1.11 & -350365 & 14909 & 0.24 \\
4 & 23998 & $2001 \mathrm{BV} 47$ & 1.18 & -437268 & 40750 & 5.66 \\
5 & 160270 & $2005 \mathrm{UP} 6$ & 0.93 & -1567067 & 43863 & 6.85 \\
6 & 84203 & $2000 \mathrm{SS} 4$ & 1.01 & -56420 & 101590 & 12.98 \\
8 & $2002 \mathrm{SF} 64$ & $2007 \mathrm{AQ} 6$ & 1.02 & -224310 & 127533 & 12.79 \\
13 & 25884 & 48527 & 1.49 & -424250 & 10616 & 1.38 \\
\hline
\end{tabular}

The case of (88259) and $1999 \mathrm{VA}_{117}$ is the one leading to the most interesting close approach. Indeed the osculating orbital elements, except the mean anomaly, of this couple are already close to the point that this was noticed and extensively discussed on the Minor Planet Mailing List in January $2008^{14}$. The time span to the close approach is so short that the nongravitational perturbations cannot change the other orbital elements other than semimajor axis and mean anomaly; both orbits are well determined (with $\operatorname{RMS}(a)<6 \times 10^{-8} \mathrm{AU}$ ), thus the nominal orbit is very close to the real orbit.

The closest approach distance we have found is not much larger than radius of the Hill's sphere of influence, which is less than $1000 \mathrm{~km}$ (depending upon the unknown density); there is no way to exclude that the encounter was in fact the escape of the smaller asteroid from an orbit around the larger

\footnotetext{
${ }^{14} \mathrm{R}$. Matson, in a MPML message of January 9, 2008 with subject Asteroid pairs: extremely close pair found gave a list of close couples including our couples 1 and 2 .
} 
one, although there is not yet enough evidence to prove this. The relative velocity is an order of magnitude less than the escape velocity (also depending upon the unknown density, but could be $1.2 \mathrm{~m} / \mathrm{s}$ ).

Still, this is just the starting point for the study of this very interesting case of asteroid couple. Non-gravitational perturbations, by using the same estimates we have used in Section 3, could change the epoch in the past at which the mean longitudes of the two asteroids are equal by several thousands of years. Although there would be anyway a very close approach at that different epoch, the fine details of the close approach, including the "true" minimum distance and relative velocity, could be different from what we find with a conservative dynamical model, including being even lower.

The couple 2, with (63440) and $2004 \mathrm{TV}_{14}$ is a difficult case because the difference in proper semimajor axis is small, to the point of being comparable to the RMS uncertainty of the osculating semimajor axis for a multiopposition asteroid; indeed, we had to update the orbit after the new observations of $2004 \mathrm{TV}_{14}$ in July 2009 to get a value of the difference in $a_{p}$ which is significant. The asteroid (63440) is currently "ahead", that is with a larger mean anomaly (by $\left.3.2^{\circ}\right)$; however, $a_{p}(63440)>a_{p}\left(2004 T V_{14}\right)$, which means that the "proper mean motion" of (63440) is slower than that of $2004 \mathrm{TV}_{14}$, and the latter should be catching up and having a close approach in the near future. A close approach in the past is possible only at an epoch far enough to allow for a full revolution of the difference in mean longitudes. Indeed, this is the case for the close approach reported in the table.

Again, the conclusion we can draw on the basis of a conservative dynamical model is that very close approaches (with low relative velocity) can take place, but this analysis is not enough to find out the actual epoch, minimum distance and relative velocity of the closest possible approach. In fact, the Yarkovsky effect, according to the order of magnitude estimates of Section 3, is in this case enough to shift a close approach in the near future to one in the near past.

The encounters found for couples 3 and 13 are also suggestive of a very slow velocity and very close encounter, although the initial orbital uncertainty and the non-gravitational perturbations may well have somewhat masked the actual values. The other 4 cases belong to the same category of couple 2 , namely, very close approaches are possible, but the actual date and properties of the closest approach cannot be established with the present analysis. 


\subsection{Possible interpretation}

An hypothesis for the interpretation of asteroid couples, very close in proper elements, has been proposed long ago, see [Milani 1994][p. 166-167], with reference to the couple of Trojan asteroids (1583) Antilochus and (3801) Thrasimedes (the distance expressed in velocity was found to be less than 10 $\mathrm{m} / \mathrm{s}$, also much less than the escape velocity). The idea, which was proposed by the late Paolo Farinella, is the following: the pairs could be obtained after an intermediate stage as binary, terminated by a low velocity escape through the so-called fuzzy boundary, generated by the heteroclinic tangle at the collinear Lagrangian points. This model predicts an escape orbit passing near one of the Lagrangian points $L_{1}$ or $L_{2}$ of the 3-body system asteroidasteroid-Sun, with a very low relative velocity of escape, which would be extremely unlikely to be obtained from a direct ejection of a splinter satellite from the larger asteroid, whatever the cause of the fission.

The evolution of the binary, in the case of the Trojans, would be dominated by tidal dissipation, and this would result in a prediction of observable properties of the pair, namely slow rotation with aligned axes, and escape velocity very close to the equator of the larger asteroid. In the case of Hungaria, which are both smaller and closer to the Sun, the non-gravitational effects such as YORP acting on the spin states and Yarkovsky acting on the orbit of the satellite could be more important. Unfortunately, a fully selfconsistent theory of the evolution of a binary asteroid taking into account the mutual orbits with full 3-body dynamics, the rotation of both objects, tidal dissipation and non-gravitational effects is not yet available ${ }^{15}$.

We do not think it is yet possible to draw firm conclusion on the origin of the close couples of Hungaria we have identified; the same problem applies to the couples of main belt and Trojan asteroids which have already been identified, and to the many more close couples which could be found by applying essentially the same methods we have used in this paper.

\subsection{Sub-families}

As shown in Figure 18, there appear to be sub-groupings in the Hungaria family, in particular at the level of distance corresponding to $40 \mathrm{~m} / \mathrm{s}$ there is no grouping including (434) Hungaria, but 4 groupings which need to be

\footnotetext{
${ }^{15}$ We are aware that there is work in progress on such topic, but nothing has been published so far.
} 
studied to assess whether they have physical significance. Of these, two merge with the main family containing (434) at the $50 \mathrm{~m} / \mathrm{s}$ level, the other two at the $60 \mathrm{~m} / \mathrm{s}$ level. This is weak evidence that the sub-groupings correspond to sub-families, that is to the outcome of a secondary collisional disruption affecting a member of the main family, and occurred much more recently than the family formation.

Of the close couples we have identified among the Hungaria, the couple number 2 including (63440) and $2004 \mathrm{TV}_{14}$ belongs to one of the subgroupings at level $40 \mathrm{~m} / \mathrm{s}$, one other object, $2004 \mathrm{TY}_{137}$ is very close (at a distance corresponding to $15 \mathrm{~m} / \mathrm{s}$ ). The couple number 4 with (23998) and $2001 \mathrm{BV}_{47}$ belongs to another sub-grouping at level $50 \mathrm{~m} / \mathrm{s}$. At the $60 \mathrm{~m} / \mathrm{s}$ level both of these couples and sub-groups merge with the main family, which at the same level includes also couples number 6 and 13, for which we have identified a possible recent split, but also couples number 7 and 10, for which we find no chance of a very close encounter in the last $2 \mathrm{My}$. This is weak evidence, which could point to a possible relationship between sub-groupings and close couples: however, the data are insufficient to discriminate between a coincidence, resulting from a presence of portions of the main family with higher number densities, and the existence of a casual relationship between secondary collisional disruption and the formation of the couples.

Thus we are convinced it is possible to draw a firm conclusion neither on the significance of the sub-groupings nor on the casual relationship with close couples. We need to point out that secondary fragmentation events, even if they could be proven, would also be likely sources of binaries, thus they would not contradict the interpretation of the close couples as obtained after an intermediate stage as binaries.

\section{Expectations for the near future}

The current level of completeness in the knowledge of Hungaria asteroids (with good orbits, that is either numbered or multi-opposition) can be assessed from the histogram of Figure 24. The largest number occurs in the bin centered at $H=16.5$. For an Hungaria favorable observing circumstances, that is observations near opposition, are for a distance from Earth of not much more than $1 \mathrm{AU}$ and from the Sun at about $2 \mathrm{AU}$, thus the apparent magnitude could be as low as $H+1.5$. This means that the well observed Hungaria have mostly been found at apparent magnitudes up to $18-19$. This value may look a bit low, since there have been asteroid surveys operat- 


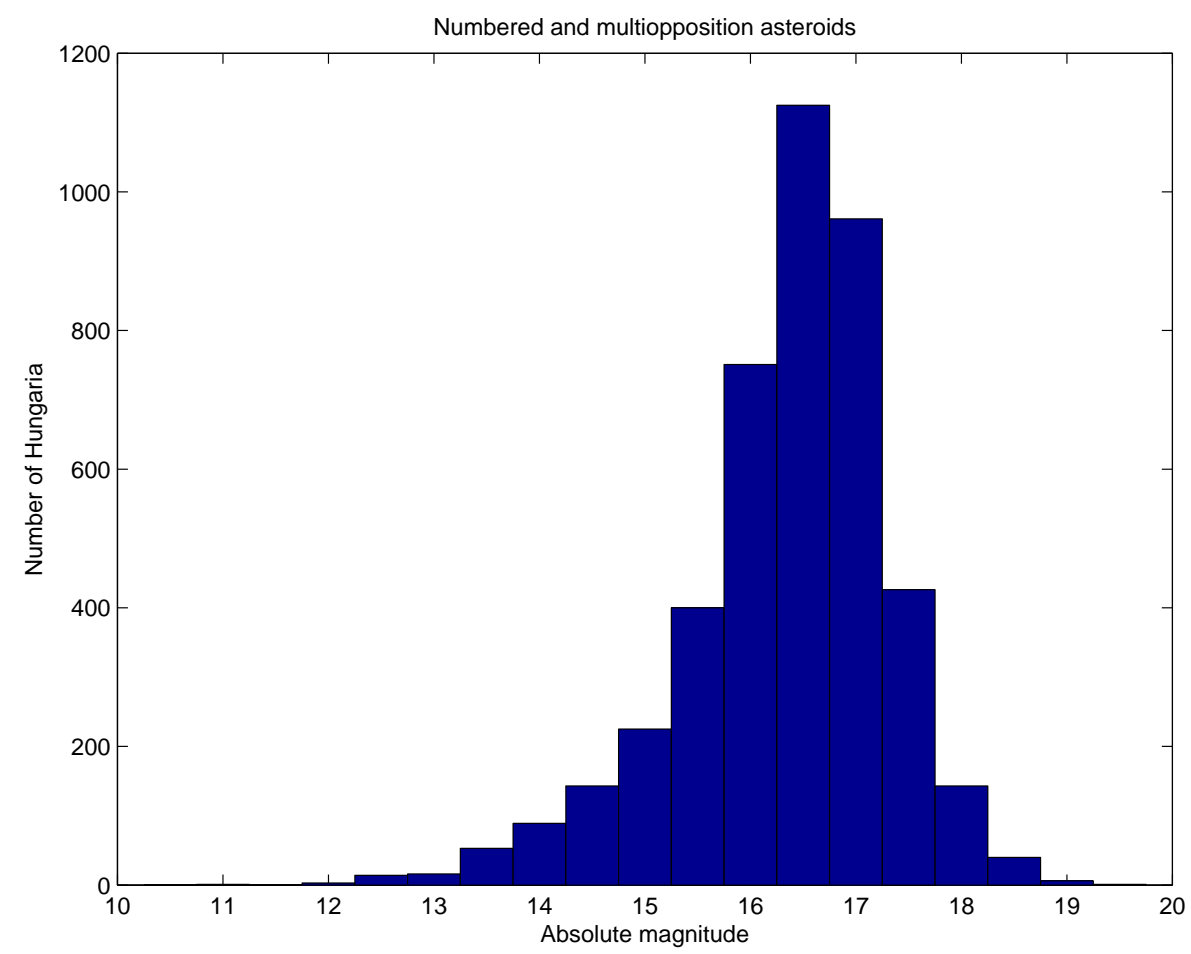

Figure 24: Histogram of the absolute magnitudes $H$ of the Hungaria asteroids in our sample with good orbits; each bin is $1 / 2$ magnitude wide.

ing with a limiting magnitude in the range $19-20$ for several years, but the Hungaria have the property of being very often, precisely near opposition, at high ecliptic latitude (up to $45^{\circ}-50^{\circ}$ ), far from the area most intensively swept by the asteroid surveys.

The next generation surveys, including Pan-STARRS (now beginning operations of the first test telescope PS1) and LSST (now polishing the primary mirror), have two properties in common: 1) the goal to achieve very high limiting magnitude, even for moving objects (nominal goals being magnitude 24 for Pan-STARRS future version PS4 and 24.5 for LSST), and 2) the ambition of covering the entire visible sky around opposition, going very far from the equator, up to the North pole for Pan-STARRS, to the South pole for LSST. They also have in common the property that these performances will not be reached tomorrow, but several years from now, taking into account fund- 
ing problems and also the unavoidable learning curve which PS1 is climbing now. Nevertheless, we can assume either one or the other of these projects, or both, will succeed within a few years from now.

The consequences of these developments will be especially dramatic in our knowledge of the Hungaria (also the Phocea and other high inclination asteroids). The $H$ magnitude of approximate completeness could peak near 21 for PS1 and later climb to beyond 22, that is we will know most of the Hungaria larger than 100 meters of diameter. Note that this implies we will know more about the Hungaria than about NEA. Although we cannot predict the number of such small Hungaria, the order of magnitude expected is such that we will know, with good orbits, many more Hungaria than the main belt asteroids we know now. Moreover, the asteroids observed with signal/noise ratio an order of magnitude above the threshold for detection, that is those up to $H=19.5$ (270 meters of diameter), will have a light-curve and multi-filter photometry accurate enough for determination of period, shape models, polar axis and color indexes. Just think to a Figure 23 extending up to $1 / D \simeq 4$ and with all objects in the plot having color and rotation data.

A research paper such as this is not the right place to anticipate the results we should be able to obtain when we will have such data. However, we think that studying the Hungaria now is a good investment of our research resources, also because of this expected future enormous data set. We also think that most of the problems for which we have not been able now to obtain a solution, or maybe only a weak one, will be fully solved.

\section{Conclusions}

We briefly summarize the firm conclusions we have been able to reach.

1. We have improved the quality of the data, by computing proper elements for thousands of Hungaria, and we will maintain an up to date catalog of such proper elements on our AstDyS site.

2. The Hungaria region is surrounded by dynamical boundaries, by which we mean that every asteroid which exits from the region for whatever reason ends up being removed by very strong dynamical effects, namely the linear secular resonances and close approaches to Mars, whose location in the phase space is well known.

3. The Hungaria region, and the Hungaria family, are crossed by nonlinear secular resonances, which result in decreased accuracy proper elements. 
They are also crossed by mean motion resonances, the most importnat being with Mars, and these can result in large changes of the proper elements $e_{p}$ and $\sin I_{p}$.

4. The non-gravitational perturbations, especially the Yarkovsky effect, have changed the semimajor axis (both osculating and proper) of the Hungaria asteroids by a large amount since the formation of the Hungaria family. This results in a large spread of all the proper elements, due to the interaction with resonances. The spread of the proper semimajor axis is asymmetric, thus pointing to a uneven distribution of spin axes, with predominant retrograde spin.

5. The Hungaria region contains a large family, called the Hungaria family because (434) Hungaria is the lowest numbered member. There is some indication of a possible second family at higher inclination.

6. The Hungaria asteroids are not all members of the Hungaria family. We have found an explicit and reliable list of these background asteroids. On the contrary, both for lack of discriminating physical observations and because of the very complicated dispersion of proper elements due to dynamical instabilities, we cannot give an explicit list of asteroids which certainly belong to the family.

7. The Hungaria group contains couples of asteroid with very close proper elements. We have identified times at which some of these couples could have had very close approaches, with low relative velocity. In some cases we have found epochs at which these encounters have taken place, within a purely conservative dynamical model. However, we believe that the Yarkovsky effect needs to be taken into account for finding the closest possible approaches.

We would like to add two comments. First, we second the appeal already contained in [Warner et al. 2009] for the largest possible set of observations of Hungaria, including astrometric follow up to improve the orbits, multicolor photometry, spectroscopy and polarimetry to identify the taxonomic types and constrain the diameters, lightcurves to find the spin rate and orientation, discoveries of binaries to constrain the masses, and if possible radar observations. This because these observations can contribute to the solution of problems which are more accessible to observational constraints in the Hungaria region, but of course are applicable to other asteroids. We think that this paper already points to some specific targets, anyway we are availble to provide further suggestions for the observers. 
Second, the progress in the understanding of the Yarkovsky effect and its consequences on the long term dynamical evolution of asteroid families appeared for some time to have decreased the value of large and accurate catalogs of proper elements. We believe we have shown in this paper that the opposite is true: because of the very interesting results which can be obtained on the Yarkovsky effects on asteroid families, very accurate proper elements are now even more useful than before.

\section{Acknowledgments}

The authors have been supported for this research by: the Italian Space Agency, under the contract ASI/INAF I/015/07/0, (A.M., A.C); the Ministry of Science and Technological Development of Serbia, under the project 146004 (Z.K. and B.N.).

\section{References}

[Bus and Binzel 2002] Bus, S.J., Binzel, R.P.: 2002, Phase II of the Small Main-Belt Asteroid Spectroscopic Survey: A Feature-Based Taxonomy. Icarus 158, 146-177.

[Carpino et al. 1987] Carpino, M., Milani, A., Nobili, A.M.: 1987, Longterm numerical integrations and synthetic theories of the motion of the outer planets. Astron. Astrophys. 181, 182-194.

[Carvano et al. 2001] Carvano, J.M. Lazzaro, D., Mothé-Diniz, T., Angeli, C.A.: 2001, Spectroscopic survey of the Hungaria and Phocea Dynamical Groups, Icarus 149, 173-189.

[Cellino et al. 1999] Cellino, A., Michel, P., Tanga, P., Zappalà, V., Paolicchi, P., Dell'Oro, A.: 1999, The velocity-size relationship for members of asteroid families and implications for the physics of catastrophic collisions. Icarus 141, 79-95.

[Cellino et al. 2002] Cellino, A., Bus, S.J., Doressoundiram, A., Lazzaro, D.: 2002. spectroscopic properties of asteroid families. In: W.F. Bottke, A. Cellino, P. Paolicchi, R.P. Binzel (eds.), Asteroids III, Univ. of Arizona Press, Tucson, pp.633-644, 
[Drummond 2000] Drummond, J.D. : 2000, The D Discriminant and NearEarth Asteroid Streams, Icarus 146, 453-475.

[Durech 2007] Durech, J., Kaasalinen, M, Warner, B.D. : 2006, New asteroid models derived from combined photometric data. Bull. Amer. Astron. Soc., 39, 468 .

[Ferraz-Mello 1981] Ferraz-Mello, S.: 1981, Estimation of periods from unequally spaced observations. Astron. J. 86, 619-624.

[Gil-Hutton et al. 2007] Gil-Hutton, R., Lazzaro, D., Benavidez, P.: 2007, Polarimetric observations of Hungaria asteroids. Astron. Astrophys. 468, 1109-1114.

[Ivezić et al. 2001] Ivezić, Ž., Tabachnik, S., Rafikov, R., et al.: 2001, Solar System Objects Observed in the Sloan Digital Sky Survey Commissioning Data. Astron. J., 122, 2749-2784.

[Knežević and Milani 2000] Knežević Z., and Milani A.: 2000 Synthetic proper elements for outer main belt asteroids. CMDA 78, 17-46.

[Knežević and Milani 2003] Knežević Z., and Milani A.: 2003, Proper element catalogs and asteroid families. Astron. Astrophys. 403, 1165-1173.

[Knežević et al. 1991] Knežević Z., Milani A., Farinella P., Froeschlè Ch., and Froeschlè C.: 1991, Secular resonances from 2 to 50 AU. Icarus 93, 316-330.

[Laskar et al. 2004] Laskar J., Robutel, P., Joutel, F., Gastineau, M., Correia, A.C.M., and Levrard, B.: 2004, A long-term numerical solution for the insolation quantities of the Earth. Astron. Astrophys. 428, 261-285.

[Lemaitre 1994] Lemaitre, A.: 1994, Hungaria: A Potential New Family, in Seventy-five years of Hirayama asteroid families: The role of collisions in the solar system history, Y. Kozai, R. P. Binzel, and T. Hirayama, Astronomical Society of the Pacific Conference Series 63, pp. 140-145.

[Lemaitre and Morbidelli 1994] Lemaitre, A., Morbidelli, A.: 1994, Proper elements for highly inclined asteroidal orbits, CMDA 60, 29-56. 
[Michel and Froeschlé 1997] Michel, P. and Froeschlè, Ch.: 1997, The location of linear secular resonances for semimajor axes smaller than $2 \mathrm{AU}$, Icarus 128, 230-240.

[Migliorini et al. 1998] Migliorini, F., Michel, P., Morbidelli, A., Nesvorný, D. and Zappalà, V.: 1998, Origin of Multikilometer Earth- and Marscrossing asteroids: a quantitative simulation, Science 281, 2022-2024.

[Milani 1994] Milani, A.: 1994, The dynamics of the Trojan asteroids. In: A. Milani, M. Di Martino and A. Cellino (eds), Asteroids, Comets, Meteors 1993, Kluwer Acad. Publ. Dordrecht, pp. 159-174.

[Milani and Knežević 1990] Milani, A., Knežević, Z.: 1990, Secular perturbation theory and the computation of asteroid proper elements, CMDA 49, 347-411.

[Milani and Knežević 1992] Milani, A., Knežević, Z.: 1992, Asteroid proper elements and secular resonances. Icarus 98, 211-232.

[Milani and Nobili 1988] Milani, A., Nobili, A. M.: 1988, Integration error over a very long time span. CMDA 43, 1-34.

[Milani and Nobili 1992] Milani, A., Nobili, A. M.: 1992, An example of the stable chaos in the Solar System. Nature, 357, 569-571.

[Milani et al. 2009] Milani, A., Chesley, S.R.,Sansaturio, M.E., Bernardi, F., Valsecchi, G.B., Arratia, O.: 2009Long-term impact risk for (101955) 1999 $\mathrm{RQ}_{36}$, Icarus, in press.

[Nesvorný et al. 2005] Nesvorný, D., Jedicke, R., Whiteley, R.J., Ivezić, Ž.: 2005, Evidence for asteroid space weathering from the Sloan Digital Sky Survey. Icarus, 173, 132-152.

[Pravec and Vokrouhlický 2009] Pravec, P., Vokrouhlick D.: 2009 Significance analysis of asteroid pairs, Icarus, in press.

[Shepard et al. 2008] Shepard, M.K., Kressler, K.M., Clark, B.E., UckertBell, M.E., Nolan, M., Howell, H.S., Mgri, C., Giorgini, J.D., Benner, L.A.M., Ostro, S.J.: 2008, Radar observations of E-class Asteroids 44 Nysa and 434 Hungaria, Icarus, 195, 220-225. 
[Vokrouhlický et al. 2000] Vokrouhlický, D., Milani A. and Chesley, S.R. 2000. Yarkovsky effect on small Near Earth asteroids: mathematical formulation and examples. Icarus 148, 118-138.

[Vokrouhlický et al. 2006a] Vokrouhlický, D., Brož, M., Morbidelli, A., Bottke, W.F., Nesvorný, A, Lazzaro, D. Rivkin, A.S.: 2006a, Yarkovsky footprints in the Eos family, Icarus 182, 92-117.

[Vokrouhlický et al. 2006b] Vokrouhlický, D., Brož, M., Bottke, W.F., Nesvorný, A, Morbidelli, A. : 2006b, Yarkovsky/YORP chronology of asteroid families, Icarus 182, 118-142.

[Warner et al. 2009] Warner, B.D., Harris, A.W., Vokrouhlick, D., Nesvorn, D., Bottke, W.F.: 2009, Analysis of the Hungaria Asteroid Population, Icarus, in press

[Zappalà et al. 1990] Zappalà, V., Cellino, A., Farinella, P., Knežević, Z.: 1990, Asteroid families. I - Identification by hierarchical clustering and reliability assessment. Astron. J., 100, 2030-2046.

[Zappalà et al. 1994] Zappalà V., Cellino, A., Farinella, P., Milani, A.: 1994, Asteroid families. II: Extension to unnumbered multiopposition asteroids. Astron. J., 107, 772-801.

[Zappalà et al. 1995] Zappalà, V., Bendjoya, Ph., Cellino, A., Farinella, P., Froeschlè, C.: 1995, Asteroid families: Search of a 12,487-asteroid sample using two different clustering techniques. Icarus, 116, 291-314. 